\title{
Observation of the Production of Three Massive Gauge Bosons at $\sqrt{s}=13 \mathrm{TeV}$
}

\author{
A. M. Sirunyan et al. ${ }^{*}$ \\ (CMS Collaboration)
}

(Received 19 June 2020; accepted 24 August 2020; published 5 October 2020)

\begin{abstract}
The first observation is reported of the combined production of three massive gauge bosons ( $V V V$ with $V=W, Z$ ) in proton-proton collisions at a center-of-mass energy of $13 \mathrm{TeV}$. The analysis is based on a data sample recorded by the CMS experiment at the CERN LHC corresponding to an integrated luminosity of $137 \mathrm{fb}^{-1}$. The searches for individual $W W W, W W Z, W Z Z$, and $Z Z Z$ production are performed in final states with three, four, five, and six leptons (electrons or muons), or with two same-sign leptons plus one or two jets. The observed (expected) significance of the combined $V V V$ production signal is 5.7 (5.9) standard deviations and the corresponding measured cross section relative to the standard model prediction is $1.02_{-0.23}^{+0.26}$. The significances of the individual $W W W$ and $W W Z$ production are 3.3 and 3.4 standard deviations, respectively. Measured production cross sections for the individual triboson processes are also reported.
\end{abstract}

DOI: 10.1103/PhysRevLett.125.151802

The production of three massive gauge bosons $V V V$ $(V=W, Z)$ in high energy proton-proton $(p p)$ collisions is interesting because the standard model (SM) predictions for these processes involve the non-Abelian character of the theory [1]. In particular, the presence of quadruple gauge boson interactions can be probed through $V V V$ production $[2,3]$. Triple gauge boson interactions and intermediate Higgs bosons $(H)$ also play a role. If physics beyond the $\mathrm{SM}$ is present at mass scales not far above $1 \mathrm{TeV}$, then cross section measurements for triple gauge boson production might deviate from SM predictions [4-7]. Up to now, such measurements have remained elusive because the production cross sections are low and backgrounds are insurmountable, except for rare leptonic final states. Next-toleading order (NLO) SM calculations predict cross sections of 509, 354, 91.6, and $37.1 \mathrm{fb}$ for $W W W, W W Z, W Z Z$, and ZZZ production at $13 \mathrm{TeV}$ with uncertainties of approximately $10 \%$ [8-12]. These calculations include contributions from the associated production of the Higgs boson with a $V$ boson, where $H$ decays to $W^{+} W^{-}$or $Z Z$ [13-16]. In this analysis, these contributions generally are not the dominant ones, even though the cross section for $\mathrm{VH}$ production is relatively large, because the event selections described below have lower acceptances for the off-shell vector boson in $H \rightarrow V V^{*}$.

This Letter reports the first observation of $V V V$ production in $p p$ collisions at $13 \mathrm{TeV}$, using a data set

\footnotetext{
*Full author list given at the end of the article.

Published by the American Physical Society under the terms of the Creative Commons Attribution 4.0 International license. Further distribution of this work must maintain attribution to the author(s) and the published article's title, journal citation, and DOI. Funded by SCOAP.
}

corresponding to an integrated luminosity of $137 \mathrm{fb}^{-1}$. Recently, the first evidence of $V V V$ production in $13 \mathrm{TeV}$ data was reported by the ATLAS Collaboration [17] following earlier searches for $W W W$ production in $8 \mathrm{TeV}$ ATLAS [18] and $13 \mathrm{TeV}$ CMS data [19]. Five final states are considered (where $\ell=e$ or $\mu$ ): $W^{ \pm} W^{ \pm} W^{\mp} \rightarrow$ $\ell^{ \pm} \ell^{ \pm} 2 \nu q \bar{q}^{\prime}, \quad W^{ \pm} W^{ \pm} W^{\mp} \rightarrow \ell^{ \pm} \ell^{ \pm} \ell^{\mp} 3 \nu, \quad W^{ \pm} W^{\mp} Z \rightarrow$ $\ell^{ \pm} \ell^{\mp} 2 \nu \ell^{ \pm} \ell^{\mp}, \quad W^{ \pm} Z Z \rightarrow \ell^{ \pm} \nu 2\left(\ell^{ \pm} \ell^{\mp}\right), \quad$ and $\quad Z Z Z \rightarrow$ $3\left(\ell^{ \pm} \ell^{\mp}\right)$. This corresponds to five exclusive channels: two same-sign (SS) leptons with jets, three $(3 \ell)$, four $(4 \ell)$, five $(5 \ell)$, and six $(6 \ell)$ leptons. Searches in the dilepton and trilepton final states target $W W W$ production; four-lepton events are used to search for $W W Z$ production; and fiveand six-lepton events are used to search for $W Z Z$ and $Z Z Z$ production, respectively.

The data were recorded in 2016-2018 with the CMS detector, whose central feature is a superconducting solenoid of $6 \mathrm{~m}$ internal diameter providing a magnetic field of 3.8 T. Within the solenoid volume are a silicon pixel and strip tracker, a lead tungstate crystal electromagnetic calorimeter, and a brass and scintillator hadron calorimeter, each composed of a barrel and two end cap sections. Forward calorimeters extend the pseudorapidity $(\eta)$ coverage provided by the barrel and end cap detectors. Muons are detected in gas-ionization chambers embedded in the steel flux-return yoke outside the solenoid. Events are selected using triggers [20] that require two electrons, two muons, or one electron and one muon passing loose isolation requirements and certain transverse momentum $\left(p_{\mathrm{T}}\right)$ thresholds. A detailed description of the detector and definitions of the coordinate system are given in Ref. [21].

The CMS event reconstruction is based on the particleflow (PF) algorithm [22], which combines information from the tracker, calorimeters, and muon systems to identify charged and neutral hadrons, photons, electrons, and muons, 
known collectively as PF candidates. Electrons and muons from $V$ decays, known as prompt leptons, are selected for off-line analysis using standard criteria [23,24]. Events containing $\tau$ leptons decaying into charged hadrons are rejected by requiring the absence of isolated tracks aside from selected electrons and muons. The PF candidates are clustered into jets using the anti- $k_{\mathrm{T}}$ algorithm with a distance parameter of 0.4 [25-27]. Jets with $p_{\mathrm{T}}>20 \mathrm{GeV}$ and $|\eta|<$ 5 are selected for the analysis. Defining the distance between a jet and a selected lepton by $\Delta R=\sqrt{(\Delta \eta)^{2}+(\Delta \phi)^{2}}$ where $\phi$ is the azimuthal angle, jets are rejected if $\Delta R<0.4$. Jets containing the decay of a $b$ quark are identified using the loose working point of the deep combined secondary-vertex $b$ tagging algorithm [28]. To increase the efficiency for identifying low- $p_{\mathrm{T}} b$ hadrons not clustered into jets, a soft $b$ tag object [29] is defined using a track-based secondary vertex reconstruction.

The primary $p p$ interaction vertex is the reconstructed vertex with the largest summed $p_{\mathrm{T}}^{2}$ calculated using trackbased jets and the associated missing transverse momentum $\left(\vec{p}_{\mathrm{T}}^{\mathrm{miss}}\right.$ ), the negative $\vec{p}_{\mathrm{T}}$ sum of those jets [30]. Track-based jets are constructed using only tracks associated with the given vertex. In addition to the primary interaction, other $p p$ interactions (pileup) produce extra charged particles and neutral energy. Only tracks associated with the primary vertex are used. The average neutral energy density from pileup is estimated, and subtracted from the reconstructed jet energies and the energy sum used in the calculation of lepton isolation [31].

The previous search for $W W W$ production [19] is based on sequences of requirements called sequential cuts. In this Letter, that approach is extended to cover all five channels. In addition, motivated by the relatively high yields in the $\mathrm{SS}, 3 \ell$, and $4 \ell$ channels, multivariate techniques based on boosted decision trees (BDTs) [32-36] are applied that outperform the sequential-cut analyses. Both the sequential-cut and BDT-based analyses are presented.

The acceptances, efficiencies, and kinematic properties of the signal and background processes are determined using a combination of data and simulated events. The POWHEG 2.0 [37-40] and the MadGraph5_aMC@ @ 2.4.2) generators [41] are used to generate $V V V$ signal events (including $V H$ ), diboson $(V V)$, and single- $t$ background events. The MadGraph5_aMC@NLO generator is used in the leading-order (LO) mode with MLM jet matching [42] to generate $\mathrm{SM} t \bar{t}, t \bar{t}+X(X=W, Z, H), W+$ jets, $Z+$ jets, $W \gamma$, and $W^{ \pm} W^{ \pm}$events. The most precise cross section calculations available are used to normalize the simulated samples, and usually correspond to either NLO or next-to-NLO accuracy $[16,41,43-50]$. Parton showering, hadronization, and the underlying event are modeled by PYTHIA (8.205 and 8.230) [51] with parameters set by the CUETP8M1 [52] and CP5 tune [53]. The NNPDF 3.0 [54] and 3.1 [55] parton distribution functions (PDFs) are used in the generation of all simulated samples. Pileup is simulated and the GEANT4 [56] package is used to mimic the response of the CMS detector.

The SS channel targets $W W W$ production [19] and requires exactly two SS leptons with $p_{\mathrm{T}}>25 \mathrm{GeV}$ and one or more jets. The dilepton mass $m_{\ell \ell}$ must exceed $20 \mathrm{GeV}$. This channel is subdivided into nine signal regions according to the flavors of the leptons $\left(e^{ \pm} e^{ \pm}, e^{ \pm} \mu^{ \pm}\right.$, or $\left.\mu^{ \pm} \mu^{ \pm}\right)$and the jet content. Events with exactly one jet are denoted " $1 J$." Events with two or more jets are categorized as " $m_{j j}$-in" or " $m_{j j}$-out" depending on whether the dijet mass for the two jets closest in $\Delta R$ is compatible with the $W$ boson mass $\left(65<m_{j j}<95 \mathrm{GeV}\right)$. The background processes fall broadly into three categories. The first category contains trilepton processes with one lepton either not selected or not reconstructed ("lost"). Such backgrounds include $W Z$ and $t \bar{t} Z$ production, which typically have only one prompt neutrino in the final state; they are reduced by requiring $m_{\mathrm{T}}^{\max }>90 \mathrm{GeV}$, where $m_{\mathrm{T}}^{\max }$ is the largest transverse mass obtained from $\vec{p}_{\mathrm{T}}^{\text {miss }}$ and any lepton in the event. The second category consists of processes with SS lepton pairs, mainly from $W^{ \pm} W^{ \pm}+$jets and $t \bar{t} W^{ \pm}$production. This contribution is suppressed by requiring $m_{J J}<$ $500 \mathrm{GeV}$ and $\left|\Delta \eta_{J J}\right|<2.5$ for the two highest- $p_{\mathrm{T}}$ jets. The third category includes $W+$ jets and $t \bar{t}+$ jets production where a final-state jet or photon is misidentified as a charged lepton, and is labeled nonprompt. These background contributions are suppressed using strict lepton identification and isolation requirements and by requiring $p_{\mathrm{T}}^{\text {miss }}>45 \mathrm{GeV}$. All backgrounds containing top quarks are further reduced by excluding events with $b$-tagged jets or soft $b$ tags. The background due to charge mismeasurement in Drell-Yan production is relevant only for dielectron events and is reduced to a negligible level by requiring $\left|m_{\ell \ell}-m_{Z}\right|>10 \mathrm{GeV}$.

The $3 \ell$ channel, which also targets $W W W$ production, is subdivided according to the number of same-flavor opposite-sign (SFOS) lepton pairs: OSFOS, 1SFOS, and 2SFOS. At least one lepton is required to have $p_{\mathrm{T}}>$ $25 \mathrm{GeV}$, while the others must have $p_{\mathrm{T}}>20 \mathrm{GeV}$, except in 0SFOS where all three leptons are required to have $p_{\mathrm{T}}>$ $25 \mathrm{GeV}$ to reduce contamination from nonprompt leptons. Events in 1 SFOS and 2SFOS must contain no jets, whereas the presence of one jet is allowed in OSFOS. The background sources are similar to those in the SS category. Events with $b$-tagged jets are excluded to suppress the nonprompt-lepton background from processes involving top quarks. The contribution from triple prompt lepton backgrounds is suppressed by requiring $\left|m_{\ell \ell}-m_{Z}\right|>20 \mathrm{GeV}$ and $m_{\ell \ell}>20 \mathrm{GeV}$ for all SFOS pairs. Additional background reduction is achieved with the following requirements: if exactly one SFOS lepton pair is found then $m_{\mathrm{T}}^{3 \mathrm{rd}}$, defined as the transverse mass calculated from $\vec{p}_{\mathrm{T}}^{\text {miss }}$ and the third lepton that is not one of the SFOS pair, must be larger than $90 \mathrm{GeV}$; and, for events with no SFOS pairs, $m_{\mathrm{T}}^{\max }>$ $90 \mathrm{GeV}$ is required. Background contributions from 
nonprompt leptons and converted or misidentified photons are reduced by requiring a large $p_{\mathrm{T}}$ of the three-lepton system $\left|\vec{p}_{\mathrm{T}}^{3 \ell}\right|>50 \mathrm{GeV}$, and a large azimuthal separation $\Delta \phi\left(\vec{p}_{\mathrm{T}}^{3 \ell}, \vec{p}_{\mathrm{T}}^{\text {miss }}\right)>2.5$ between $\vec{p}_{\mathrm{T}}^{\text {miss }}$ and $\vec{p}_{\mathrm{T}}^{3 \ell}$. Events with a conversion photon emitted in a $Z$ boson decay are suppressed by requiring $\left|m_{3 \ell}-m_{Z}\right|>10 \mathrm{GeV}$ where $m_{3 \ell}$ is the three-lepton invariant mass.

The $4 \ell$ channel targets $W W Z$ production. The $Z$ boson is identified through its decay to a SFOS lepton pair with $\left|m_{\ell \ell}-m_{Z}\right|<10 \mathrm{GeV}$. These leptons are required to have $p_{\mathrm{T}}>25(10) \mathrm{GeV}$ for the (sub)leading lepton. The (sub) leading lepton of the remaining non- $Z$ leptons must have $p_{\mathrm{T}}>25(10) \mathrm{GeV}$. The dominant background comes from $Z Z$ production, so the cases of different-flavor $(e \mu)$ and same-flavor $(e e / \mu \mu)$ non- $Z$ lepton pairs are handled separately. The non- $Z$ same-flavor invariant mass is required to differ from $m_{Z}$ by at least $10 \mathrm{GeV}$. Other background contributions consist of $t \bar{t} Z, t W Z, t \bar{t} H$, and $W Z$ events. The rejection of events with $b$-tagged jets reduces contributions from top quarks and a requirement that $m_{\ell \ell}>12 \mathrm{GeV}$ for all opposite-sign lepton pairs suppresses backgrounds from low-mass resonances. The $4 \ell$ channel is subdivided into seven signal regions: for the $e \mu$ category there are four bins in $m_{\ell \ell}$ and $m_{\mathrm{T} 2}$ [57], and for the $e e / \mu \mu$ category there are three bins based on $p_{\mathrm{T}}^{4 \ell}$ and $p_{\mathrm{T}}^{\text {miss }}$.

The $5 \ell$ and $6 \ell$ channels target $W Z Z$ and $Z Z Z$ production, respectively. Event yields are low because of small cross sections and branching fractions. Since background contributions are low, the selection maximizes the signal efficiency. The two leading leptons are required to have $p_{\mathrm{T}}>25 \mathrm{GeV}$ and other leptons must have $p_{\mathrm{T}}>10 \mathrm{GeV}$. Events in the $5 \ell$ channel are required to contain two SFOS lepton pairs with $\left|m_{\ell \ell}-m_{Z}\right|<15 \mathrm{GeV}$. The background in the $5 \ell$ channel consists almost entirely of $Z Z$ events with a nonprompt lepton, which is usually an electron. The background is reduced by requiring $m_{\mathrm{T}}>50 \mathrm{GeV}$, where $m_{\mathrm{T}}$ is calculated from $\vec{p}_{\mathrm{T}}^{\mathrm{miss}}$ and that electron. Smaller background contributions arise from $t \bar{t} Z$ and $t \bar{t} H$ production, which are reduced by rejecting events with $b$-tagged jets. Events in the $6 \ell$ channel are required to have three SFOS pairs and a sixlepton scalar $p_{\mathrm{T}}$ sum larger than $250 \mathrm{GeV}$. The small $6 \ell$ background comes from $t \bar{t} H$ and $Z Z$ production.

Background contributions from sources with a particular number of prompt leptons and no nonprompt leptons in signal regions are estimated using simulations with correction factors, typically near unity, derived from several control regions in data enriched in the main sources of background events. Both the predicted numbers of events and relevant kinematic distributions are compared with observations in control regions to derive the correction factors. The precision of the comparison is used to assess systematic uncertainties in these background contributions. Background contributions from sources with one or more nonprompt leptons cannot be reliably evaluated using simulations, so estimates based on control samples in data are used instead. These estimates rely on the fact that nonprompt leptons tend to be less isolated than prompt ones. For the SS and $3 \ell$ channels, following Ref. [19], the contribution of events with a nonprompt lepton is evaluated using a sample of events in which one lepton satisfies loose identification criteria but fails the tight criteria. The number of events in this region determines the estimate of the nonprompt background in the signal region using a transfer factor computed with a separate event sample rich in nonprompt leptons. This transfer factor is the ratio of the number of events that pass the tight selection criteria to those that pass the loose criteria. For the $5 \ell$ channel, a sample of events with three prompt leptons and one nonprompt lepton is dominated by $W Z$ production and used to verify the prediction of background contributions with nonprompt leptons. Nonprompt leptons are a minor background for all other channels.

The signal strength $\mu$, defined as the measured production cross section times branching fraction divided by the expected SM value, is determined through simultaneous fits to all twenty-one signal regions. In one version of the fit, four independent signal strengths $\left(\mu_{W W W}, \mu_{W W Z}, \mu_{W Z Z}\right.$, and $\left.\mu_{Z Z Z}\right)$ are used. In the other version, a common signal strength $\mu_{\text {comb }}$ is used for all four processes.

The most important sources of systematic uncertainty involve the estimation of background contributions; the uncertainties range from 5\% to $25 \%$ and come mainly from limited statistical precision in the control regions. The uncertainties in the nonprompt background estimates from control samples in data also contribute significantly at $50 \%$. Uncertainties related to trigger efficiencies, lepton identification and energy resolution, jet energy scale, and $b$-jet tagging efficiency range from $1 \%$ to $9 \%$. A $2.3 \%-2.5 \%$ uncertainty in the integrated luminosity is assessed [58-60]. Uncertainties due to limitations of the theory include missing higher-order corrections (2\%-14\%), PDF uncertainties (2\%-7\%), and the strong coupling $\alpha_{\mathrm{S}}(1 \%)$. Theoretical and experimental uncertainties are correlated across different channels. Statistical uncertainties are much larger than systematic ones. The expected significance of the combined $V V V$ production signal based on the sequentialcut selection is 5.4 standard deviations (s.d.), and the observed significance is 5.0 s.d. The observed (expected) significances for the individual triboson production processes are 2.5 (2.9) s.d. for $W W W, 3.5$ (3.6) s.d. for $W W Z$, $1.6(0.7)$ s.d. for $W Z Z$, and $0.0(0.9)$ s.d. for $Z Z Z$.

The discrimination of signal and background events in the SS, $3 \ell$, and $4 \ell$ channels is enhanced by using BDTs. The training and optimization of the BDTs is carried out for each channel using simulated background and signal events. A minimum value of each BDT output variable substitutes for the categorizations of events and the kinematic requirements applied in the sequential-cut analyses. In the SS and $3 \ell$ channels, two separate BDTs are trained: the first one to separate the signal from the nonprompt 


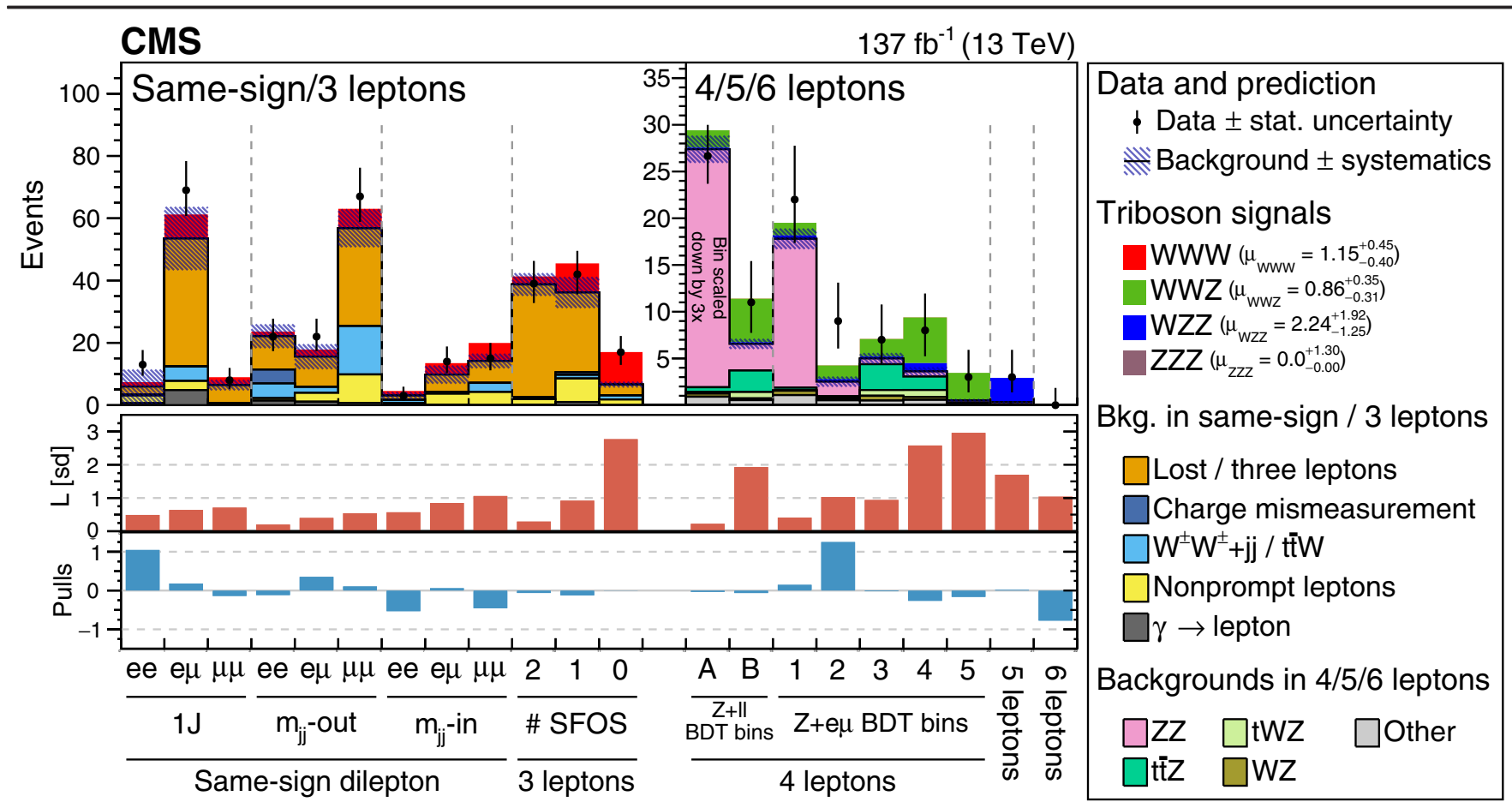

FIG. 1. Comparison of the observed numbers of events to the predicted yields after fitting. For the $W W W$ and $W W Z$ channels, the results from the BDT-based selections are used. The $V V V$ signal is shown stacked on top of the total background. The points represent the data and the error bars show the statistical uncertainties. The expected significance $L$ in the middle panel represents the number of standard deviations (s.d.) with which the null hypothesis (no signal) is rejected; it is calculated for the fit for $\mu_{\text {comb. The lower panel }}$ shows the pulls for the fit result.

background and the second one to separate the signal from the rest of the background. These two BDTs are applied sequentially. In the $4 \ell$ channel, a similar strategy is pursued except that the two BDTs are targeted against $Z Z$ and $t t Z$ backgrounds specifically. There are two (five) signal regions for events in the $e e / \mu \mu(e \mu)$ category. The improvement in sensitivity due to the use of BDTs varies channel by channel and is in the range 5\%-15\%. No BDTs are used for the $5 \ell$ and $6 \ell$ channels.

The yields in the individual signal regions obtained using the BDTs are shown in Fig. 1. The significances $L$ of the expected numbers of events are computed including systematic uncertainties and are evaluated under the asymptotic approximation [61]. Pulls are the differences in the numbers of observed and predicted events normalized to the uncertainties in the numbers of predicted events. Assuming the SM production of $V V V$ events, the expected significance of the fit with a single signal strength $\mu_{\text {comb }}$ is 5.9 s.d. and the observed significance is 5.7 s.d. The observed (expected) significances for the individual triboson production processes are $3.3(3.1)$ s.d. for $W W W$, $3.4(4.1)$ s.d. for $W W Z, 1.7(0.7)$ s.d. for $W Z Z$, and $0.0(0.9)$ s.d. for $Z Z Z$. In the most sensitive signal regions, approximately one third of the $V V V$ events come from $V H$ production. The measured signal strengths, obtained in the asymptotic approximation of the $\mathrm{CL}_{\mathrm{s}}$ method [61], correspond to the total cross sections listed in Table I; leptonic branching fractions for $W$ and $Z$ decays come from Ref. [62]. If $V H$ is considered as a background, then the combined observed (expected) significance for $\mu_{\text {comb }}$ is 2.9 (3.5) s.d. and the measured cross sections are listed in Table I. For $Z Z Z$ production, upper limits are reported at 95\% confidence level. Signal strengths obtained using both sequential-cut and BDT-based approaches and with $\mathrm{VH}$ production counted as signal are summarized in Fig. 2.

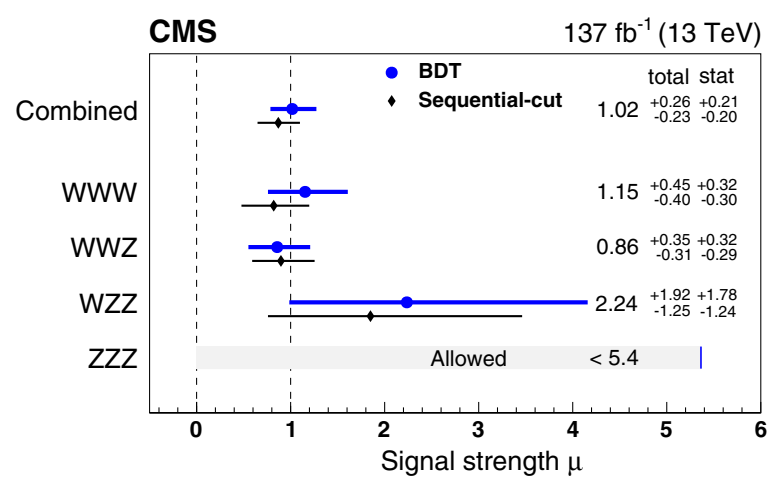

FIG. 2. Best fit values of the signal strengths for the BDT-based analyses (blue solid circles) and the sequential-cut analyses (black solid diamonds). The error bars represent the total uncertainty. For $Z Z Z$ production, a 95\% confidence level upper limit is shown. The stated numerical values correspond to the BDT-based analysis. 
TABLE I. Measured cross sections obtained with the BDTbased analyses. The uncertainties listed are statistical and systematic. For the results listed in the middle (right) column of the table, Higgs boson contributions are counted as signal (background). The $V V V$ cross section is calculated from the fit for $\mu_{\text {comb }}$. For $Z Z Z$ production, 95\% confidence level upper limits are reported.

\begin{tabular}{lcc}
\hline \hline & \multicolumn{2}{c}{ Cross section $(\mathrm{fb})$} \\
\cline { 2 - 3 } & \multicolumn{2}{c}{ Treating Higgs boson contributions as } \\
\cline { 2 - 3 } Process & Signal & Background \\
\hline$V V V$ & $1010_{-200-120}^{+210+150}$ & $370_{-130-60}^{+140+80}$ \\
$W W W$ & $590_{-150-130}^{+160+160}$ & $190_{-100-70}^{+110+80}$ \\
$W W Z$ & $300_{-100-40}^{+120+50}$ & $100_{-70-30}^{+80+30}$ \\
$W Z Z$ & $200_{-110+20}^{+160+70}$ & $110_{-70}^{+100+30}$ \\
$Z Z Z$ & $<200$ & $<80$ \\
\hline \hline
\end{tabular}

In summary, proton-proton collision data at $\sqrt{s}=$ $13 \mathrm{TeV}$ recorded with the CMS experiment and amounting to $137 \mathrm{fb}^{-1}$ were used to observe the production of three massive gauge bosons. The significance of the observation is 5.7 standard deviations (s.d.) with 5.9 s.d. expected. For $W W W(W W Z$ ) production, the observed significance is 3.3 (3.4) s.d. compatible with 3.1 (4.1) s.d. expected. Measured cross sections for $W W W, W W Z$, and $W Z Z$ production and an upper limit for $Z Z Z$ production are in agreement with the expectations of the standard model. This Letter documents the evidence for $W W W$ and $W W Z$ production and the first observation of the combined production of three massive gauge bosons.

We thank our colleagues in the CERN accelerator departments for the excellent performance of the LHC and thank the technical and administrative staffs at CERN and at other CMS institutes for their contributions to the success of the CMS effort. In addition, we gratefully acknowledge the computing centers and personnel of the Worldwide LHC Computing Grid for delivering so effectively the computing infrastructure essential to our analyses. Finally, we acknowledge the enduring support for the construction and operation of the LHC and the CMS detector provided by the following funding agencies: BMBWF and FWF (Austria); FNRS and FWO (Belgium); CNPq, CAPES, FAPERJ, FAPERGS, and FAPESP (Brazil); MES (Bulgaria); CERN; CAS, MoST, and NSFC (China); COLCIENCIAS (Colombia); MSES and CSF (Croatia); RIF (Cyprus); SENESCYT (Ecuador); MoER, ERC IUT, PUT and ERDF (Estonia); Academy of Finland, MEC, and HIP (Finland); CEA and CNRS/IN2P3 (France); BMBF, DFG, and HGF (Germany); GSRT (Greece); NKFIA (Hungary); DAE and DST (India); IPM (Iran); SFI (Ireland); INFN (Italy); MSIP and NRF (Republic of Korea); MES (Latvia); LAS (Lithuania); MOE and UM (Malaysia); BUAP, CINVESTAV, CONACYT, LNS, SEP, and UASLP-FAI (Mexico); MOS
(Montenegro); MBIE (New Zealand); PAEC (Pakistan); MSHE and NSC (Poland); FCT (Portugal); JINR (Dubna); MON, RosAtom, RAS, RFBR, and NRC KI (Russia); MESTD (Serbia); SEIDI, CPAN, PCTI, and FEDER (Spain); MOSTR (Sri Lanka); Swiss Funding Agencies (Switzerland); MST (Taipei); ThEPCenter, IPST, STAR, and NSTDA (Thailand); TUBITAK and TAEK (Turkey); NASU (Ukraine); STFC (United Kingdom); DOE and NSF (USA).

[1] P. Langacker, Tests of the standard model and searches for new physics, Adv. Ser. Dir. High Energy Phys. 14, 883 (1995).

[2] G. Bélanger and F. Boudjema, Probing quartic couplings of weak bosons through three vectors production at a $500 \mathrm{GeV}$ NLC, Phys. Lett. B 288, 201 (1992).

[3] S. Godfrey, Quartic gauge boson couplings, AIP Conf. Proc. 350, 209 (1995).

[4] W. Buchmüller and D. Wyler, Effective Lagrangian analysis of new interactions and flavor conservation, Nucl. Phys. B268, 621 (1986).

[5] O. J. P. Eboli, M. C. Gonzalez-Garcia, and S. M. Lietti, Bosonic quartic couplings at CERN LHC, Phys. Rev. D 69, 095005 (2004).

[6] C. Degrande, N. Greiner, W. Kilian, O. Mattelaer, H. Mebane, T. Stelzer, S. Willenbrock, and C. Zhang, Effective field theory: A modern approach to anomalous couplings, Ann. Phys. (Amsterdam) 335, 21 (2013).

[7] C. Degrande, A basis of dimension-eight operators for anomalous neutral triple gauge boson interactions, J. High Energy Phys. 02 (2014) 101.

[8] A. Lazopoulos, K. Melnikov, and F. Petriello, QCD corrections to triboson production, Phys. Rev. D 76, 014001 (2007).

[9] T. Binoth, G. Ossola, C. G. Papadopoulos, and R. Pittau, NLO QCD corrections to triboson production, J. High Energy Phys. 06 (2008) 082.

[10] V. Hankele and D. Zeppenfeld, QCD corrections to hadronic $W W Z$ production with leptonic decays, Phys. Lett. B 661 , 103 (2008).

[11] F. Campanario, V. Hankele, C. Oleari, S. Prestel, and D. Zeppenfeld, QCD corrections to charged triple vector boson production with leptonic decay, Phys. Rev. D 78, 094012 (2008)

[12] S. Dittmaier, A. Huss, and G. Knippen, Next-to-leadingorder QCD and electroweak corrections to $W W W$ production at proton-proton colliders, J. High Energy Phys. 09 (2017) 034.

[13] T. Han and S. Willenbrock, QCD correction to the $p p \rightarrow$ WH and $\mathrm{ZH}$ total cross-sections, Phys. Lett. B 273, 167 (1991).

[14] O. Brein, A. Djouadi, and R. Harlander, NNLO QCD corrections to the Higgs-strahlung processes at hadron colliders, Phys. Lett. B 579, 149 (2004).

[15] M. L. Ciccolini, S. Dittmaier, and M. Kramer, Electroweak radiative corrections to associated $\mathrm{WH}$ and $\mathrm{ZH}$ production at hadron colliders, Phys. Rev. D 68, 073003 (2003). 
[16] D. de Florian et al., Handbook of LHC Higgs cross sections: 4. Deciphering the nature of the Higgs sector, CERN Report No. CERN-2017-002-M, 2016.

[17] ATLAS Collaboration, Evidence for the production of three massive vector bosons with the ATLAS detector, Phys. Lett. B 798, 134913 (2019).

[18] ATLAS Collaboration, Search for triboson $W^{ \pm} W^{ \pm} W^{\mp}$ production in $p p$ collisions at $\sqrt{s}=8 \mathrm{TeV}$ with the ATLAS detector, Eur. Phys. J. C 77, 141 (2017).

[19] CMS Collaboration, Search for the production of $W^{ \pm} W^{ \pm} W^{\mp}$ events at $\sqrt{s}=13 \mathrm{TeV}$, Phys. Rev. D 100, 012004 (2019).

[20] CMS Collaboration, The CMS trigger system, J. Instrum. 12, P01020 (2017).

[21] CMS Collaboration, The CMS experiment at the CERN LHC, J. Instrum. 3, S08004 (2008).

[22] CMS Collaboration, Particle-flow reconstruction and global event description with the CMS detector, J. Instrum. 12, P10003 (2017).

[23] CMS Collaboration, Performance of electron reconstruction and selection with the CMS detector in protonproton collisions at $\sqrt{s}=8 \mathrm{TeV}$, J. Instrum. 10, P06005 (2015).

[24] CMS Collaboration, Performance of the CMS muon detector and muon reconstruction with proton-proton collisions at $\sqrt{s}=13 \mathrm{TeV}$, J. Instrum. 13, P06015 (2018).

[25] M. Cacciari and G. P. Salam, Dispelling the $N^{3}$ myth for the $k_{\mathrm{T}}$ jet-finder, Phys. Lett. B 641, 57 (2006).

[26] M. Cacciari, G. P. Salam, and G. Soyez, The anti- $k_{\mathrm{T}}$ jet clustering algorithm, J. High Energy Phys. 04 (2008) 063.

[27] M. Cacciari, G. P. Salam, and G. Soyez, FastJet user manual, Eur. Phys. J. C 72, 1896 (2012).

[28] CMS Collaboration, Identification of heavy-flavour jets with the CMS detector in $p p$ collisions at $13 \mathrm{TeV}$, J. Instrum. 13, P05011 (2018).

[29] CMS Collaboration, Search for direct production of supersymmetric partners of the top quark in the all-jets final state in proton-proton collisions at $\sqrt{s}=13 \mathrm{TeV}$, J. High Energy Phys. 10 (2017) 005.

[30] CMS Collaboration, Technical proposal for the Phase-II upgrade of the Compact Muon Solenoid, CMS Technical proposal Report No. CERN-LHCC-2015-010, CMS-TDR15-02, 2015.

[31] M. Cacciari and G. P. Salam, Pileup subtraction using jet areas, Phys. Lett. B 659, 119 (2008).

[32] L. Breiman, J. Friedman, R. A. Olshen, and C. J. Stone, Classification and Regression Trees (Chapman and Hall/CRC, Boca Raton, 1984).

[33] I. Narsky, Optimization of signal significance by bagging decision trees, in Statistical Problems in Particle Physics, Astrophysics and Cosmology (PHYSTAT 05): Proceedings, Oxford, UK, 2005 (World Scientific, Singapore, 2005), p. 143, http://dx.doi.org/10.1142/9781860948985_0030.

[34] B. P. Roe, H.-J. Yang, J. Zhu, Y. Liu, I. Stancu, and G. McGregor, Boosted decision trees, an alternative to artificial neural networks, Nucl. Instrum. Methods Phys. Res., Sect. A 543, 577 (2005).

[35] CMS Collaboration, Search for $s$ channel single top quark production in pp collisions at $\sqrt{s}=7$ and $8 \mathrm{TeV}$, J. High Energy Phys. 09 (2016) 027.
[36] T. Chen and C. Guestrin, Xgboost: A scalable tree boosting system, in Proceedings of the 22nd SACM SIGKDD International Conference on Knowledge Discovery and Data Mining (ACM, San Francisco, 2016), p. 785.

[37] P. Nason, A new method for combining NLO QCD with shower Monte Carlo algorithms, J. High Energy Phys. 11 (2004) 040.

[38] S. Frixione, P. Nason, and C. Oleari, Matching NLO QCD computations with parton shower simulations: The POWHEG method, J. High Energy Phys. 11 (2007) 070.

[39] S. Alioli, P. Nason, C. Oleari, and E. Re, A general framework for implementing NLO calculations in shower Monte Carlo programs: The POWHEG BOX, J. High Energy Phys. 06 (2010) 043.

[40] E. Re, Single-top $W t$-channel production matched with parton showers using the POWHEG method, Eur. Phys. J. C 71, 1547 (2011).

[41] J. Alwall, R. Frederix, S. Frixione, V. Hirschi, F. Maltoni, O. Mattelaer, H. S. Shao, T. Stelzer, P. Torrielli, and M. Zaro, The automated computation of tree-level and next-to-leading order differential cross sections, and their matching to parton shower simulations, J. High Energy Phys. 07 (2014) 079.

[42] J. Alwall, S. Hoeche, F. Krauss, N. Lavesson, L. Lonnblad, F. Maltoni, M. L. Mangano, M. Moretti, C. G. Papadopoulos, F. Piccinini, S. Schumann, M. Treccani, J. Winter, and M. Worek, Comparative study of various algorithms for the merging of parton showers and matrix elements in hadronic collisions, Eur. Phys. J. C 53, 473 (2008).

[43] Y. Li and F. Petriello, Combining QCD and electroweak corrections to dilepton production in FEWZ, Phys. Rev. D 86, 094034 (2012).

[44] J. M. Campbell, R. K. Ellis, and C. Williams, Vector boson pair production at the LHC, J. High Energy Phys. 07 (2011) 018.

[45] M. Czakon and A. Mitov, TOP++: A program for the calculation of the top-pair cross-section at hadron colliders, Comput. Phys. Commun. 185, 2930 (2014).

[46] R. Gavin, Y. Li, F. Petriello, and S. Quackenbush, $W$ physics at the LHC with FEWZ 2.1, Comput. Phys. Commun. 184, 209 (2013).

[47] J. M. Campbell and R. K. Ellis, $t \bar{t} W^{ \pm}$production and decay at NLO, J. High Energy Phys. 07 (2012) 052.

[48] M. V. Garzelli, A. Kardos, C. G. Papadopoulos, and Z. Trocsanyi, $t \bar{t} W^{ \pm}$and $t \bar{t} Z$ hadroproduction at NLO accuracy in QCD with parton shower and hadronization effects, J. High Energy Phys. 11 (2012) 056.

[49] F. Cascioli, T. Gehrmann, M. Grazzini, S. Kallweit, P. Maierhöfer, A. von Manteuffel, S. Pozzorini, D. Rathlev, L. Tancredi, and E. Weihs, ZZ production at hadron colliders in NNLO QCD, Phys. Lett. B 735, 311 (2014).

[50] M. Grazzini, S. Kallweit, D. Rathlev, and M. Wiesemann, $W^{ \pm} Z$ production at hadron colliders in NNLO QCD, Phys. Lett. B 761, 179 (2016).

[51] T. Sjöstrand, S. Ask, J. R. Christiansen, R. Corke, N. Desai, P. Ilten, S. Mrenna, S. Prestel, C. O. Rasmussen, and P. Z. Skands, An introduction to PYTHIA 8.2, Comput. Phys. Commun. 191, 159 (2015).

[52] CMS Collaboration, Event generator tunes obtained from underlying event and multiparton scattering measurements, Eur. Phys. J. C 76, 155 (2016). 
[53] CMS Collaboration, Extraction and validation of a new set of CMS PYTHIA8 tunes from underlying-event measurements, Eur. Phys. J. C 80, 4 (2020).

[54] R. D. Ball et al. (NNPDF Collaboration), Parton distributions for the LHC Run II, J. High Energy Phys. 04 (2015) 040.

[55] R. D. Ball et al. (NNPDF Collaboration), Parton distributions from high-precision collider data, Eur. Phys. J. C 77, 663 (2017).

[56] S. Agostinelli et al. (GEANT4 Collaboration), GEANT4-A simulation toolkit, Nucl. Instrum. Methods Phys. Res., Sect. A 506, 250 (2003).

[57] C. G. Lester and D. J. Summers, Measuring masses of semiinvisibly decaying particle pairs produced at hadron colliders, Phys. Lett. B 463, 99 (1999).

[58] CMS Collaboration, CMS luminosity measurements for the 2016 data taking period, CMS Physics Analysis Summary
CMS-PAS-LUM-17-001， 2016， http://cds.cern.ch/record/ 2621960.

[59] CMS Collaboration, CMS luminosity measurements for the 2017 data taking period at $\sqrt{s}=13 \mathrm{TeV}$, CMS Physics Analysis Summary CMS-PAS-LUM-17-004, 2017, http:// cds.cern.ch/record/2621960.

[60] CMS Collaboration, CMS luminosity measurements for the 2018 data taking period at $\sqrt{s}=13 \mathrm{TeV}, \mathrm{CMS}$ Physics Analysis Summary No. CMS-PAS-LUM-18-002, 2018, http://cds.cern.ch/record/2676164.

[61] G. Cowan, K. Cranmer, E. Gross, and O. Vitells, Asymptotic formulae for likelihood-based tests of new physics, Eur. Phys. J. C 71, 1554 (2011); Erratum, Eur. Phys. J. C 73, 2501 (2013).

[62] M. Tanabashi et al. (Particle Data Group), Review of particle physics, Phys. Rev. D 98, 030001 (2018).

A. M. Sirunyan, ${ }^{1, a}$ A. Tumasyan, ${ }^{1}$ W. Adam, ${ }^{2}$ F. Ambrogi,${ }^{2}$ T. Bergauer, ${ }^{2}$ M. Dragicevic, ${ }^{2}$ J. Erö, ${ }^{2}$ A. Escalante Del Valle, ${ }^{2}$ R. Frühwirth, ${ }^{2, b}$ M. Jeitler, ${ }^{2, b}$ N. Krammer, ${ }^{2}$ L. Lechner, ${ }^{2}$ D. Liko, ${ }^{2}$ T. Madlener, ${ }^{2}$ I. Mikulec, ${ }^{2}$ F. M. Pitters, ${ }^{2}$ N. Rad, ${ }^{2}$ J. Schieck, ${ }^{2, b}$ R. Schöfbeck, ${ }^{2}$ M. Spanring, ${ }^{2}$ S. Templ, ${ }^{2}$ W. Waltenberger, ${ }^{2}$ C.-E. Wulz, ${ }^{2, b}$ M. Zarucki, ${ }^{2}$ V. Chekhovsky, ${ }^{3}$ A. Litomin, ${ }^{3}$ V. Makarenko, ${ }^{3}$ J. Suarez Gonzalez, ${ }^{3}$ M. R. Darwish,${ }^{4, c}$ E. A. De Wolf, ${ }^{4}$ D. Di Croce, ${ }^{4}$ X. Janssen, ${ }^{4}$ T. Kello, ${ }^{4, d}$ A. Lelek, ${ }^{4}$ M. Pieters, ${ }^{4}$ H. Rejeb Sfar ${ }^{4}$ H. Van Haevermaet, ${ }^{4}$ P. Van Mechelen, ${ }^{4}$ S. Van Putte, ${ }^{4}$ N. Van Remortel, ${ }^{4}$ F. Blekman, ${ }^{5}$ E. S. Bols, ${ }^{5}$ S. S. Chhibra, ${ }^{5}$ J. D'Hondt, ${ }^{5}$ J. De Clercq, ${ }^{5}$ D. Lontkovskyi, ${ }^{5}$ S. Lowette, ${ }^{5}$ I. Marchesini, ${ }^{5}$ S. Moortgat, ${ }^{5}$ A. Morton, ${ }^{5}$ Q. Python, ${ }^{5}$ S. Tavernier, ${ }^{5}$ W. Van Doninck,${ }^{5}$ P. Van Mulders, ${ }^{5}$ D. Beghin, ${ }^{6}$ B. Bilin, ${ }^{6}$ B. Clerbaux, ${ }^{6}$ G. De Lentdecker, ${ }^{6}$ H. Delannoy, ${ }_{6}^{6}$ B. Dorney, ${ }_{6}^{6}$ L. Favart, ${ }^{6}$ A. Grebenyuk, ${ }^{6}$ A. K. Kalsi, ${ }^{6}{ }^{6}$. Makarenko, ${ }^{6}$ L. Moureaux, ${ }^{6}$ L. Pétré, ${ }^{6}$ A. Popov, ${ }^{6}$ N. Postiau, ${ }^{6}$ E. Starling, ${ }^{6}$ L. Thomas, ${ }^{6}$ C. Vander Velde, ${ }^{6}$ P. Vanlaer, ${ }^{6}$ D. Vannerom, ${ }^{6}$ L. Wezenbeek, ${ }^{6}$ T. Cornelis, ${ }^{7}$ D. Dobur, ${ }^{7}$ M. Gruchala, ${ }^{7}$ I. Khvastunov, ${ }^{7, e}$ M. Niedziela, ${ }^{7}$ C. Roskas, ${ }^{7}$ K. Skovpen, ${ }^{7}$ M. Tytgat, ${ }^{7}$ W. Verbeke, ${ }^{7}$ B. Vermassen, ${ }^{7}$ M. Vit, ${ }^{7}$ G. Bruno, ${ }^{8}$ F. Bury, ${ }^{8}$ C. Caputo, ${ }^{8}$ P. David, ${ }^{8}$ C. Delaere, ${ }^{8}$ M. Delcourt, ${ }^{8}$ I. S. Donertas, ${ }^{8}$ A. Giammanco, ${ }^{8}$ V. Lemaitre, ${ }^{8}$ K. Mondal, ${ }^{8}$ J. Prisciandaro, ${ }^{8}$ A. Taliercio, ${ }^{8}$ M. Teklishyn,${ }^{8}$ P. Vischia, ${ }^{8}$ S. Wuyckens, ${ }^{8}$ J. Zobec ${ }^{8}$ G. A. Alves, ${ }^{9}$ G. Correia Silva, ${ }^{9}$ C. Hensel,${ }^{9}$ A. Moraes, ${ }^{9}$ W. L. Aldá Júnior, ${ }^{10}$

E. Belchior Batista Das Chagas, ${ }^{10}$ H. Brandao Malbouisson, ${ }^{10}$ W. Carvalho, ${ }^{10}$ J. Chinellato, ${ }^{10, f}$ E. Coelho, ${ }^{10}$

E. M. Da Costa, ${ }^{10}$ G. G. Da Silveira ${ }^{10, g}$ D. De Jesus Damiao, ${ }^{10}$ S. Fonseca De Souza, ${ }^{10}$ J. Martins, ${ }^{10, h}$

D. Matos Figueiredo, ${ }^{10}$ M. Medina Jaime, ${ }^{10, \mathrm{i}}$ M. Melo De Almeida ${ }^{10}$ C. Mora Herrera, ${ }^{10}$ L. Mundim,${ }^{10}$ H. Nogima,${ }^{10}$

P. Rebello Teles, ${ }^{10}$ L. J. Sanchez Rosas, ${ }^{10}$ A. Santoro, ${ }^{10}$ S. M. Silva Do Amaral, ${ }^{10}$ A. Sznajder, ${ }^{10}$ M. Thiel, ${ }^{10}$

E. J. Tonelli Manganote, ${ }^{10, f}$ F. Torres Da Silva De Araujo, ${ }^{10}$ A. Vilela Pereira, ${ }^{10}$ C. A. Bernardes,${ }^{11 a}$ L. Calligaris,${ }^{11 a}$

T. R. Fernandez Perez Tomei, ${ }^{11 \mathrm{a}}$ E. M. Gregores,${ }^{11 \mathrm{a}, 11 \mathrm{~b}}$ D. S. Lemos, ${ }^{11 \mathrm{a}}$ P. G. Mercadante, ${ }^{11 \mathrm{a}, 11 \mathrm{~b}}$ S. F. Novaes, ${ }^{1 \mathrm{a}}$

Sandra S. Padula, ${ }^{11 a}$ A. Aleksandrov, ${ }^{12}$ G. Antchev, ${ }^{12}$ I. Atanasov, ${ }^{12}$ R. Hadjiiska, ${ }^{12}$ P. Iaydjiev, ${ }^{12}$ M. Misheva, ${ }^{12}$ M. Rodozov, ${ }^{12}$ M. Shopova, ${ }^{12}$ G. Sultanov, ${ }^{12}$ M. Bonchev, ${ }^{13}$ A. Dimitrov, ${ }^{13}$ T. Ivanov, ${ }^{13}$ L. Litov, ${ }^{13}$ B. Pavlov,${ }^{13}$ P. Petkov, ${ }^{13}$ A. Petrov, ${ }^{13}$ W. Fang, ${ }^{14, d}$ Q. Guo, ${ }^{14}$ H. Wang, ${ }^{14}$ L. Yuan, ${ }^{14}$ M. Ahmad,${ }^{15}$ Z. Hu, ${ }^{15}$ Y. Wang, ${ }^{15}$ E. Chapon,${ }^{16}$ G. M. Chen, ${ }^{16, j}$ H. S. Chen, ${ }^{16, j}$ M. Chen, ${ }^{16}$ D. Leggat,${ }^{16}$ H. Liao, ${ }^{16}$ Z. Liu, ${ }^{16}$ R. Sharma, ${ }^{16}$ A. Spiezia, ${ }^{16}$ J. Tao, ${ }^{16}$ J. Thomas-wilsker, ${ }^{16}$ J. Wang, ${ }^{16}$ H. Zhang, ${ }^{16}$ S. Zhang, ${ }^{16, j}$ J. Zhao, ${ }^{16}$ A. Agapitos, ${ }^{17}$ Y. Ban, ${ }^{17}$ C. Chen, ${ }^{17}$ A. Levin, ${ }^{17}$ J. Li, ${ }^{17}$ Q. Li,${ }^{17}$ M. Lu, ${ }^{17}$ X. Lyu, ${ }^{17}$ Y. Mao,${ }^{17}$ S. J. Qian, ${ }^{17}$ D. Wang,,${ }^{17}$ Q. Wang,${ }^{17}$ J. Xiao,${ }^{17}$ Z. You, ${ }^{18}$ X. Gao, ${ }^{19, d}$ M. Xiao,${ }^{20}$ C. Avila,${ }^{21}$ A. Cabrera, ${ }^{21}$ C. Florez ${ }^{21}$ J. Fraga, ${ }^{21}$ A. Sarkar, ${ }^{21}$ M. A. Segura Delgado, ${ }^{21}$ J. Jaramillo, ${ }^{22}$ J. Mejia Guisao, ${ }^{22}$ F. Ramirez, ${ }^{22}$

J. D. Ruiz Alvarez, ${ }^{22}$ C. A. Salazar González, ${ }^{22}$ N. Vanegas Arbelaez, ${ }^{22}$ D. Giljanovic, ${ }^{23}$ N. Godinovic,${ }^{23}$ D. Lelas, ${ }^{23}$ I. Puljak, ${ }^{23}$ T. Sculac, ${ }^{23}$ Z. Antunovic, ${ }^{24}$ M. Kovac, ${ }^{24}$ V. Brigljevic, ${ }^{25}$ D. Ferencek, ${ }^{25}$ D. Majumder, ${ }^{25}$ B. Mesic, ${ }^{25}$ M. Roguljic, ${ }^{25}$ A. Starodumov, ${ }^{25, k}$ T. Susa, ${ }^{25}$ M. W. Ather, ${ }^{26}$ A. Attikis, ${ }^{26}$ E. Erodotou, ${ }^{26}$ A. Ioannou, ${ }^{26}$ G. Kole, ${ }^{26}$ M. Kolosova, ${ }^{26}$ S. Konstantinou, ${ }^{26}$ G. Mavromanolakis, ${ }^{26}$ J. Mousa, ${ }^{26}$ C. Nicolaou, ${ }^{26}$ F. Ptochos, ${ }^{26}$ P. A. Razis, ${ }^{26}$ H. Rykaczewski, ${ }^{26}$ H. Saka, ${ }^{26}$ D. Tsiakkouri, ${ }^{26}$ M. Finger, ${ }^{27,1}$ M. Finger Jr., ${ }^{27,1}$ A. Kveton, ${ }^{27}$ J. Tomsa, ${ }^{27}$ E. Ayala, ${ }^{28}$ E. Carrera Jarrin, ${ }^{29}$ S. Abu Zeid ${ }^{30}$ S. Khalil, ${ }^{30, m}$ E. Salama, ${ }^{30, n, o}$ A. Lotfy, ${ }^{31}$ M. A. Mahmoud, ${ }^{31}$ S. Bhowmik, ${ }^{32}$ A. Carvalho Antunes De Oliveira, ${ }^{32}$ R. K. Dewanjee, ${ }^{32}$ K. Ehataht, ${ }^{32}$ M. Kadastik, ${ }^{32}$ M. Raidal, ${ }^{32}$ C. Veelken, ${ }^{32}$ P. Eerola, ${ }^{33}$ 
L. Forthomme, ${ }^{33}$ H. Kirschenmann, ${ }^{33}$ K. Osterberg, ${ }^{33}$ M. Voutilainen, ${ }^{33}$ E. Brücken, ${ }^{34}$ F. Garcia, ${ }^{34}$ J. Havukainen, ${ }^{34}$ V. Karimäki, ${ }^{34}$ M. S. Kim, ${ }^{34}$ R. Kinnunen, ${ }^{34}$ T. Lampén, ${ }^{34}$ K. Lassila-Perini, ${ }^{34}$ S. Laurila, ${ }^{34}$ S. Lehti, ${ }^{34}$ T. Lindén, ${ }^{34}$ H. Siikonen, ${ }^{34}$ E. Tuominen, ${ }^{34}$ J. Tuominiemi, ${ }^{34}$ P. Luukka, ${ }^{35}$ T. Tuuva, ${ }^{35}$ M. Besancon, ${ }^{36}$ F. Couderc, ${ }^{36}$ M. Dejardin, ${ }^{36}$ D. Denegri, ${ }^{36}$ J. L. Faure,${ }^{36}$ F. Ferri, ${ }^{36}$ S. Ganjour,${ }^{36}$ A. Givernaud,${ }^{36}$ P. Gras,${ }^{36}$ G. Hamel de Monchenault, ${ }^{36}$ P. Jarry, ${ }^{36}$ B. Lenzi ${ }^{36}$ E. Locci, ${ }^{36}$ J. Malcles, ${ }^{36}$ J. Rander, ${ }^{36}$ A. Rosowsky, ${ }^{36}$ M. Ö. Sahin, ${ }^{36}$ A. Savoy-Navarro, ${ }^{36, p}$ M. Titov, ${ }^{36}$ G. B. Yu, ${ }^{36}$ S. Ahuja, ${ }^{37}$ C. Amendola,${ }^{37}$ F. Beaudette, ${ }^{37}$ M. Bonanomi, ${ }^{37}$ P. Busson,${ }^{37}$ C. Charlot,${ }^{37}$ O. Davignon, ${ }^{37}$ B. Diab,${ }^{37}$ G. Falmagne, ${ }^{37}$ R. Granier de Cassagnac, ${ }^{37}$ I. Kucher, ${ }^{37}$ A. Lobanov, ${ }^{37}$ C. Martin Perez, ${ }^{37}$ M. Nguyen, ${ }^{37}$ C. Ochando, ${ }^{37}$ P. Paganini, ${ }^{37}$ J. Rembser, ${ }^{37}$ R. Salerno, ${ }^{37}$ J. B. Sauvan, ${ }^{37}$ Y. Sirois, ${ }^{37}$ A. Zabi, ${ }^{37}$ A. Zghiche, ${ }^{37}$ J.-L. Agram, ${ }^{38, q}$ J. Andrea ${ }^{38}$ D. Bloch, ${ }^{38}$ G. Bourgatte, ${ }^{38}$ J.-M. Brom, ${ }^{38}$ E. C. Chabert, ${ }^{38}$ C. Collard, ${ }^{38}$ J.-C. Fontaine, ${ }^{38, q}$ D. Gelé, ${ }^{38}$ U. Goerlach, ${ }^{38}$ C. Grimault ${ }^{38}$ A.-C. Le Bihan, ${ }^{38}$ P. Van Hove, ${ }^{38}$ E. Asilar, ${ }^{39}$ S. Beauceron, ${ }^{39}$ C. Bernet, ${ }^{39}$ G. Boudoul, ${ }^{39}$ C. Camen,${ }^{39}$ A. Carle ${ }^{39}$ N. Chanon, ${ }^{39}$ D. Contardo, ${ }^{39}$ P. Depasse, ${ }^{39}$ H. El Mamouni, ${ }^{39}$ J. Fay,${ }^{39}$ S. Gascon, ${ }^{39}$ M. Gouzevitch, ${ }^{39}$ B. Ille,${ }^{39}$ Sa. Jain, ${ }^{39}$ I. B. Laktineh, ${ }^{39}$ H. Lattaud, ${ }^{39}$ A. Lesauvage, ${ }^{39}$ M. Lethuillier, ${ }^{39}$ L. Mirabito,${ }^{39}$ L. Torterotot, ${ }^{39}$ G. Touquet,${ }^{39}$ M. Vander Donckt, ${ }^{39}$ S. Viret, ${ }^{39}$ T. Toriashvili, ${ }^{40, r}$ Z. Tsamalaidze, ${ }^{40,1}$ L. Feld, ${ }^{41}$ K. Klein, ${ }^{41}$ M. Lipinski, ${ }^{41}$ D. Meuser, ${ }^{41}$ A. Pauls, ${ }^{41}$ M. Preuten, ${ }^{41}$ M. P. Rauch,${ }^{41}$ J. Schulz,${ }^{41}$ M. Teroerde, ${ }^{41}$ D. Eliseev, ${ }^{42}$ M. Erdmann,${ }^{42}$ P. Fackeldey, ${ }^{42}$ B. Fischer, ${ }^{42}$ S. Ghosh, ${ }^{42}$ T. Hebbeker, ${ }^{42}$ K. Hoepfner, ${ }^{42}$ H. Keller,${ }^{42}$ L. Mastrolorenzo, ${ }^{42}$ M. Merschmeyer, ${ }^{42}$ A. Meyer,${ }^{42}$ P. Millet, ${ }^{42}$ G. Mocellin, ${ }^{42}$ S. Mondal, ${ }^{42}$ S. Mukherjee, ${ }^{42}$ D. Noll, ${ }^{42}$ A. Novak,${ }^{42}$ T. Pook, ${ }^{42}$ A. Pozdnyakov, ${ }^{42}$ T. Quast, ${ }^{42}$ M. Radziej, ${ }^{42}$ Y. Rath, ${ }^{42}$ H. Reithler, ${ }^{42}$ J. Roemer, ${ }^{42}$ A. Schmidt, ${ }^{42}$ S. C. Schuler, ${ }^{42}$ A. Sharma,${ }^{42}$ S. Wiedenbeck, ${ }^{42}$ S. Zaleski, ${ }^{42}$ C. Dziwok, ${ }^{43}$ G. Flügge, ${ }^{43}$ W. Haj Ahmad ${ }^{43, s}$ O. Hlushchenko, ${ }^{43}$ T. Kress ${ }^{43}$ A. Nowack,${ }^{43}$ C. Pistone, ${ }^{43}$ O. Pooth, ${ }^{43}$ D. Roy, ${ }^{43}$ H. Sert, ${ }^{43}$ A. Stahl,${ }^{43, t}$ T. Ziemons, ${ }^{43}$ H. Aarup Petersen, ${ }^{44}$ M. Aldaya Martin, ${ }^{44}$ P. Asmuss, ${ }^{44}$ I. Babounikau, ${ }^{44}$ S. Baxter, ${ }^{44}$ O. Behnke, ${ }^{44}$ A. Bermúdez Martínez, ${ }^{44}$ A. A. Bin Anuar, ${ }^{44}$ K. Borras, ${ }^{44, u}$ V. Botta, ${ }^{44}$ D. Brunner, ${ }^{44}$ A. Campbell, ${ }^{44}$ A. Cardini, ${ }^{44}$ P. Connor, ${ }^{44}$ S. Consuegra Rodríguez, ${ }^{44}$ V. Danilov, ${ }^{44}$ A. De Wit,${ }^{44}$ M. M. Defranchis, ${ }^{44}$ L. Didukh, ${ }^{44}$ D. Domínguez Damiani,${ }^{44}$ G. Eckerlin, ${ }^{44}$ D. Eckstein, ${ }^{44}$ T. Eichhorn,${ }^{44}$ A. Elwood ${ }^{44}$ L. I. Estevez Banos, ${ }^{44}$ E. Gallo, ${ }^{44, v}$ A. Geiser ${ }^{44}$ A. Giraldi, ${ }^{44}$ A. Grohsjean, ${ }^{44}$ M. Guthoff, ${ }^{44}$ A. Harb, ${ }^{44}$ A. Jafari, ${ }^{44, w}$ N. Z. Jomhari, ${ }^{44}$ H. Jung, ${ }^{44}$ A. Kasem, ${ }^{44, u}$ M. Kasemann, ${ }^{44}$ H. Kaveh, ${ }^{44}$ J. Keaveney, ${ }^{44}$ C. Kleinwort, ${ }^{44}$ J. Knolle, ${ }^{44}$ D. Krücker, ${ }^{44}$ W. Lange, ${ }^{44}$ T. Lenz, ${ }^{44}$ J. Lidrych ${ }^{44}$ K. Lipka, ${ }^{44}$ W. Lohmann, ${ }^{44, x}$ R. Mankel, ${ }^{44}$ I.-A. Melzer-Pellmann, ${ }^{44}$ J. Metwally, ${ }^{44}$ A. B. Meyer, ${ }^{44}$ M. Meyer, ${ }^{44}$ M. Missiroli, ${ }^{44}$ J. Mnich, ${ }^{44}$ A. Mussgiller, ${ }^{44}$ V. Myronenko, ${ }^{44}$ Y. Otarid, ${ }^{44}$ D. Pérez Adán, ${ }^{44}$ S. K. Pflitsch, ${ }^{44}$ D. Pitzl, ${ }^{44}$ A. Raspereza, ${ }^{44}$ A. Saggio, ${ }^{44}$ A. Saibel,${ }^{44}$ M. Savitskyi, ${ }^{44}$ V. Scheurer ${ }^{44}$

P. Schütze, ${ }^{44}$ C. Schwanenberger,${ }^{44}$ R. Shevchenko, ${ }^{44}$ A. Singh,${ }^{44}$ R. E. Sosa Ricardo ${ }^{44}$ H. Tholen, ${ }^{44}$ N. Tonon, ${ }^{44}$ O. Turkot, ${ }^{44}$ A. Vagnerini, ${ }^{44}$ M. Van De Klundert, ${ }^{44}$ R. Walsh,${ }^{44}$ D. Walter ${ }^{44}$ Y. Wen, ${ }^{44}$ K. Wichmann, ${ }^{44}$ C. Wissing, ${ }^{44}$ S. Wuchterl, ${ }^{44}$ O. Zenaiev, ${ }^{44}$ R. Zlebcik, ${ }^{44}$ R. Aggleton, ${ }^{45}$ S. Bein, ${ }^{45}$ L. Benato, ${ }^{45}$ A. Benecke, ${ }^{45}$ K. De Leo, ${ }^{45}$ T. Dreyer ${ }^{45}$ A. Ebrahimi, ${ }^{45}$ F. Feindt, ${ }^{45}$ A. Fröhlich, ${ }^{45}$ C. Garbers, ${ }^{45}$ E. Garutti, ${ }^{45}$ D. Gonzalez, ${ }^{45}$ P. Gunnellini, ${ }^{45}$ J. Haller, ${ }^{45}$ A. Hinzmann, ${ }^{45}$ A. Karavdina, ${ }^{45}$ G. Kasieczka, ${ }^{45}$ R. Klanner, ${ }^{45}$ R. Kogler, ${ }^{45}$ S. Kurz, ${ }^{45}$ V. Kutzner, ${ }^{45}$ J. Lange, ${ }^{45}$ T. Lange ${ }^{45}$ A. Malara, ${ }^{45}$ J. Multhaup,${ }^{45}$ C. E. N. Niemeyer, ${ }^{45}$ A. Nigamova, ${ }^{45}$ K. J. Pena Rodriguez, ${ }^{45}$ O. Rieger,${ }^{45}$ P. Schleper,${ }^{45}$ S. Schumann, ${ }^{45}$ J. Schwandt, ${ }^{45}$ D. Schwarz, ${ }^{45}$ J. Sonneveld, ${ }^{45}$ H. Stadie,${ }^{45}$ G. Steinbrück, ${ }^{45}$ B. Vormwald, ${ }^{45}$ I. Zoi, ${ }^{45}$ M. Baselga, ${ }^{46}$ S. Baur, ${ }^{46}$ J. Bechtel, ${ }^{46}$ T. Berger,${ }^{46}$ E. Butz, ${ }^{46}$ R. Caspart ${ }^{46}$ T. Chwalek, ${ }^{46}$ W. De Boer,${ }^{46}$ A. Dierlamm, ${ }^{46}$ A. Droll,${ }^{46}$ K. El Morabit, ${ }^{46}$ N. Faltermann, ${ }^{46}$ K. Flöh, ${ }^{46}$ M. Giffels, ${ }^{46}$ A. Gottmann, ${ }^{46}$ F. Hartmann, ${ }^{46, t}$ C. Heidecker, ${ }^{46}$ U. Husemann, ${ }^{46}$ M. A. Iqbal, ${ }^{46}$ I. Katkov, ${ }^{46, y}$ P. Keicher, ${ }^{46}$ R. Koppenhöfer, ${ }^{46}$ S. Kudella, ${ }^{46}$ S. Maier ${ }^{46}$ M. Metzler, ${ }^{46}$ S. Mitra, ${ }^{46}$ M. U. Mozer, ${ }^{46}$ D. Müller, ${ }^{46}$ Th. Müller, ${ }^{46}$ M. Musich ${ }^{46}$ G. Quast, ${ }^{46}$ K. Rabbertz, ${ }^{46}$ J. Rauser, ${ }^{46}$ D. Savoiu, ${ }^{46}$ D. Schäfer, ${ }^{46}$ M. Schnepf ${ }^{46}$ M. Schröder, ${ }^{46}$ D. Seith, ${ }^{46}$ I. Shvetsov, ${ }^{46}$ H. J. Simonis, ${ }^{46}$ R. Ulrich, ${ }^{46}$ M. Wassmer, ${ }^{46}$ M. Weber, ${ }^{46}$ C. Wöhrmann, ${ }^{46}$ R. Wolf, ${ }^{46}$ S. Wozniewski, ${ }^{46}$ G. Anagnostou, ${ }^{47}$ P. Asenov, ${ }^{47}$ G. Daskalakis,${ }^{47}$ T. Geralis,${ }^{47}$ A. Kyriakis, ${ }^{47}$ D. Loukas, ${ }^{47}$ G. Paspalaki, ${ }^{47}$ A. Stakia, ${ }^{47}$ M. Diamantopoulou, ${ }^{48}$ D. Karasavvas,${ }^{48}$ G. Karathanasis, ${ }^{48}$ P. Kontaxakis, ${ }^{48}$ C. K. Koraka, ${ }^{48}$ A. Manousakis-katsikakis, ${ }^{48}$ A. Panagiotou, ${ }^{48}$ I. Papavergou, ${ }^{48}$ N. Saoulidou, ${ }^{48}$

K. Theofilatos, ${ }^{48}$ K. Vellidis, ${ }^{48}$ E. Vourliotis ${ }^{48}$ G. Bakas,${ }^{49}$ K. Kousouris,${ }^{49}$ I. Papakrivopoulos, ${ }^{49}$ G. Tsipolitis, ${ }^{49}$ A. Zacharopoulou, ${ }^{49}$ I. Evangelou, ${ }^{50}$ C. Foudas, ${ }^{50}$ P. Gianneios,${ }^{50}$ P. Katsoulis,${ }^{50}$ P. Kokkas ${ }^{50}$ S. Mallios ${ }^{50}$ K. Manitara, ${ }^{50}$ N. Manthos, ${ }^{50}$ I. Papadopoulos,${ }^{50}$ J. Strologas ${ }^{50}$ M. Bartók, ${ }^{51, z}$ R. Chudasama, ${ }^{51}$ M. Csanad ${ }^{51}$ M. M. A. Gadallah, ${ }^{51, a a}$ S. Lökös, ${ }^{51, b b}$ P. Major, ${ }^{51}$ K. Mandal,${ }^{51}$ A. Mehta, ${ }^{51}$ G. Pasztor, ${ }^{51}$ O. Surányi,${ }^{51}$ G. I. Veres,${ }^{51}$ G. Bencze, ${ }^{52}$ C. Hajdu, ${ }^{52}$ D. Horvath, ${ }^{52, c \mathrm{cc}}$ F. Sikler, ${ }^{52}$ V. Veszpremi, ${ }^{52}$ G. Vesztergombi, ${ }^{52, \mathrm{a}, \mathrm{bb}}$ S. Czellar, ${ }^{53}$ J. Karancsi, ${ }^{53, \mathrm{z}}$ J. Molnar, ${ }^{53}$ Z. Szillasi, ${ }^{53}$ D. Teyssier, ${ }^{53}$ P. Raics ${ }^{54}$ Z. L. Trocsanyi, ${ }^{54}$ B. Ujvari, ${ }^{54}$ T. Csorgo,${ }^{55}$ F. Nemes,${ }^{55}$ T. Novak, ${ }^{55}$ S. Choudhury, ${ }^{56}$ J. R. Komaragiri, ${ }^{56}$ D. Kumar, ${ }^{56}$ L. Panwar, ${ }^{56}$ P. C. Tiwari, ${ }^{56}$ S. Bahinipati, ${ }^{57, d d}$ D. Dash, ${ }^{57}$ C. Kar, ${ }^{57}$ P. Mal, ${ }^{57}$ T. Mishra, ${ }^{57}$ 
V. K. Muraleedharan Nair Bindhu, ${ }^{57}$ A. Nayak,${ }^{57, \text { ee }}$ D. K. Sahoo, ${ }^{57, d d}$ N. Sur, ${ }^{57}$ S. K. Swain, ${ }^{57}$ S. Bansal, ${ }^{58}$ S. B. Beri, ${ }^{58}$ V. Bhatnagar, ${ }^{58}$ S. Chauhan, ${ }^{58}$ N. Dhingra, ${ }^{58, f f}$ R. Gupta, ${ }^{58}$ A. Kaur,${ }^{58}$ A. Kaur, ${ }^{58}$ S. Kaur, ${ }^{58}$ P. Kumari, ${ }^{58}$ M. Lohan, ${ }^{58}$ M. Meena ${ }^{58}$ K. Sandeep, ${ }^{58}$ S. Sharma, ${ }^{58}$ J. B. Singh, ${ }^{58}$ A. K. Virdi, ${ }^{58}$ A. Ahmed, ${ }^{59}$ A. Bhardwaj, ${ }^{59}$ B. C. Choudhary, ${ }^{59}$ R. B. Garg, ${ }^{59}$ M. Gola,${ }^{59}$ S. Keshri, ${ }^{59}$ A. Kumar, ${ }^{59}$ M. Naimuddin, ${ }^{59}$ P. Priyanka, ${ }^{59}$ K. Ranjan, ${ }^{59}$ A. Shah,${ }^{59}$ M. Bharti, ${ }^{60, g g}$ R. Bhattacharya, ${ }^{60}$ S. Bhattacharya,${ }^{60}$ D. Bhowmik,${ }^{60}$ S. Dutta, ${ }^{60}$ S. Ghosh ${ }^{60}$ B. Gomber,${ }^{60, \text { hh }}$ M. Maity, ${ }^{60, \text { ii }}$ S. Nandan,${ }^{60}$ P. Palit, ${ }^{60}$ A. Purohit ${ }^{60}$ P. K. Rout, ${ }^{60}$ G. Saha ${ }^{60}$ S. Sarkar, ${ }^{60}$ M. Sharan, ${ }^{60}$ B. Singh, ${ }^{60, g g}$ S. Thakur, ${ }^{60, g g}$ P. K. Behera, ${ }^{61}$ S. C. Behera, ${ }^{61}$ P. Kalbhor, ${ }^{61}$ A. Muhammad ${ }^{61}$ R. Pradhan,${ }^{61}$ P. R. Pujahari, ${ }^{61}$ A. Sharma, ${ }^{61}$ A. K. Sikdar ${ }^{61}$ D. Dutta, ${ }^{62}$ V. Jha, ${ }^{62}$ V. Kumar, ${ }^{62}$ D. K. Mishra, ${ }^{62}$ K. Naskar, ${ }^{62, j j}$ P. K. Netrakanti, ${ }^{62}$ L. M. Pant, ${ }^{62}$ P. Shukla, ${ }^{62}$ T. Aziz, ${ }^{63}$ M. A. Bhat, ${ }^{63}$ S. Dugad, ${ }^{63}$ R. Kumar Verma, ${ }^{63}$ U. Sarkar, ${ }^{63}$ S. Banerjee, ${ }^{64}$ S. Bhattacharya ${ }^{64}$ S. Chatterjee, ${ }^{64}$ P. Das, ${ }^{64}$ M. Guchait, ${ }^{64}$ S. Karmakar, ${ }^{64}$ S. Kumar, ${ }^{64}$ G. Majumder,${ }^{64}$ K. Mazumdar,${ }^{64}$ S. Mukherjee, ${ }^{64}$ D. Roy,${ }^{64}$ N. Sahoo,${ }^{64}$ S. Dube,${ }^{65}$ B. Kansal, ${ }^{65}$ A. Kapoor, ${ }^{65}$ K. Kothekar, ${ }^{65}$ S. Pandey, ${ }^{65}$ A. Rane, ${ }^{65}$ A. Rastogi, ${ }^{65}$ S. Sharma, ${ }^{65}$ H. Bakhshiansohi, ${ }^{66, k k}$ S. Chenarani, ${ }^{67,11}$ S. M. Etesami, ${ }^{67}$ M. Khakzad ${ }^{67}$ M. Mohammadi Najafabadi, ${ }^{67}$ M. Naseri, ${ }^{67}$ M. Felcini ${ }^{68}$ M. Grunewald ${ }^{68}$ M. Abbrescia, ${ }^{69 a, 69 b}$ R. Aly ${ }^{69 a, 69 b, m m}$ C. Aruta, ${ }^{69 a, 69 b}$ A. Colaleo, ${ }^{69 a}$ D. Creanza, ${ }^{69 a, 69 c}$ N. De Filippis, ${ }^{69 a, 69 c}$ M. De Palma ${ }^{69 a, 69 b}$ A. Di Florio, ${ }^{69 a, 69 b}$ A. Di Pilato, ${ }^{69 a, 69 b}$ W. Elmetenawee, ${ }^{69 a, 69 b}$ L. Fiore, ${ }^{69 a}$ A. Gelmi, ${ }^{69,69 b}$ M. Gul, ${ }^{69 a}$ G. Iaselli, ${ }^{69 a, 69 c}$ M. Ince, ${ }^{69 a, 69 b}$ S. Lezki,${ }^{69 a, 69 b}$ G. Maggi, ${ }^{69 a, 69 c}$ M. Maggi, ${ }^{69 a}$ I. Margjeka, ${ }^{69 a, 69 b}$ J. A. Merlin, ${ }^{69 a}$ S. My, ${ }^{69 a, 69 b}$ S. Nuzzo, ${ }^{69 a, 69 b}$ A. Pompili, ${ }^{69 a, 69 b}$ G. Pugliese ${ }^{69 a, 69 c}$ G. Selvaggi, ${ }^{69 a, 69 b}$ L. Silvestris, ${ }^{69 a}$ F. M. Simone,${ }^{69 a, 69 b}$ R. Venditti, ${ }^{69 a}$ P. Verwilligen, ${ }^{69 \mathrm{a}}$ G. Abbiendi, ${ }^{70 \mathrm{a}}$ C. Battilana,${ }^{70 a, 70 b}$ D. Bonacorsi, ${ }^{70 a, 70 b}$ L. Borgonovi, ${ }^{70 a, 70 b}$ S. Braibant-Giacomelli, ${ }^{70 a, 70 b}$ L. Brigliadori, ${ }^{70 a, 70 b}$ R. Campanini, ${ }^{70 a, 70 b}$ P. Capiluppi, ${ }^{70 a, 70 b}$ A. Castro, ${ }^{70 a}, 70 \mathrm{~b}$ F. R. Cavallo, ${ }^{70 a}$ C. Ciocca, ${ }^{70 a}$ M. Cuffiani, ${ }^{70 a, 70 b}$ G. M. Dallavalle, ${ }^{70 a}$ T. Diotalevi, ${ }^{70 a, 70 b}$ F. Fabbri, ${ }^{70 a}$ A. Fanfani, ${ }^{70 a, 70 b}$ E. Fontanesi, ${ }^{70 a, 70 b}$ P. Giacomelli, ${ }^{70 a}$ C. Grandi, ${ }^{70 a}$ L. Guiducci, ${ }^{70 a, 70 b}$ F. Iemmi, ${ }^{70 a, 70 b}$ S. Lo Meo, ${ }^{70 a n n}$ S. Marcellini, ${ }^{70 a}$ G. Masetti, ${ }^{70 a}$ F. L. Navarria, ${ }^{70 a, 70 b}$ A. Perrotta, ${ }^{70 a}$ F. Primavera, ${ }^{70 a, 70 b}$ T. Rovelli, ${ }^{70 a, 70 b}$ G. P. Siroli,${ }^{70 a, 70 b}$ N. Tosi, ${ }^{70 a}$ S. Albergo, ${ }^{71 a, 71 b, o o}$ S. Costa, ${ }^{71 a, 71 b}$ A. Di Mattia, ${ }^{71 a}$ R. Potenza, ${ }^{71 a, 71 b}$ A. Tricomi, ${ }^{71 a, 71 b, 0 o}$ C. Tuve, ${ }^{71 a, 71 b}$ G. Barbagli, ${ }^{72 a}$ A. Cassese, ${ }^{72 a}$ R. Ceccarelli ${ }^{72 a, 72 b}$ V. Ciulli, ${ }^{72 a, 72 b}$ C. Civinini, ${ }^{72 a}$ R. D’Alessandro, ${ }^{72 a, 72 b}$ F. Fiori, ${ }^{72 a}$ E. Focardi, ${ }^{72 a, 72 b}$ G. Latino, ${ }^{72 a, 72 b}$ P. Lenzi, ${ }^{72 a, 72 b}$ M. Lizzo, ${ }^{72 a, 72 b}$ M. Meschini, ${ }^{72 a}$ S. Paoletti, ${ }^{72 a}$ R. Seidita ${ }^{72 a, 72 b}$ G. Sguazzoni, ${ }^{72 a}$ L. Viliani, ${ }^{72 a}$ L. Benussi,${ }^{73}$ S. Bianco, ${ }^{73}$ D. Piccolo, ${ }^{73}$ M. Bozzo, ${ }^{74 a, 74 b}$ F. Ferro, ${ }^{74 a}$ R. Mulargia, ${ }^{74 a, 74 b}$ E. Robutti ${ }^{74 a}$ S. Tosi, ${ }^{74 a, 74 b}$ A. Benaglia, ${ }^{75 a}$ A. Beschi, ${ }^{75 a, 75 b}$ F. Brivio, ${ }^{75 a, 75 b}$ F. Cetorelli, ${ }^{75 a, 75 b}$ V. Ciriolo, ${ }^{75 a, 75 b, t}$ F. De Guio, ${ }^{75 a, 75 b}$ M. E. Dinardo, ${ }^{75 a, 75 b}$ P. Dini, ${ }^{75 a}$ S. Gennai, ${ }^{75 a}$ A. Ghezzi, ${ }^{75 a, 75 b}$ P. Govoni, ${ }^{75 a, 75 b}$ L. Guzzi, ${ }^{75 a, 75 b}$ M. Malberti, ${ }^{75 a}$ S. Malvezzi,${ }^{75 a}$ D. Menasce, ${ }^{75 a}$ F. Monti, ${ }^{75 a, 75 b}$

L. Moroni ${ }^{75 a}$ M. Paganoni, ${ }^{75 a, 75 b}$ D. Pedrini, ${ }^{75 a}$ S. Ragazzi, ${ }^{75 a, 75 b}$ T. Tabarelli de Fatis, ${ }^{75 a, 75 b}$ D. Valsecchi, ${ }^{75 a, 75 b, t}$ D. Zuolo, ${ }^{75 a, 75 b}$ S. Buontempo, ${ }^{76 a}$ N. Cavallo, ${ }^{76 a, 76 c}$ A. De Iorio,${ }^{76 a, 76 b}$ F. Fabozzi, ${ }^{76 a, 76 c}$ F. Fienga, ${ }^{76 a}$ A. O. M. Iorio, ${ }^{76 a, 76 b}$ L. Layer, ${ }^{76 a, 76 b}$ L. Lista, ${ }^{76 a, 76 b}$ S. Meola ${ }^{76 a, 76 d, t}$ P. Paolucci, ${ }^{76 a, t}$ B. Rossi, ${ }^{76 a}$ C. Sciacca, ${ }^{76 a, 76 b}$ E. Voevodina, ${ }^{76 a, 76 b}$ P. Azzi, ${ }^{77 a}$ N. Bacchetta, ${ }^{77 a}$ D. Bisello, ${ }^{77,77 b}$ A. Boletti, ${ }^{77 a, 77 b}$ A. Bragagnolo, ${ }^{77 a, 77 b}$ R. Carlin, ${ }^{77 a, 77 b}$ P. Checchia, ${ }^{77 a}$

P. De Castro Manzano, ${ }^{77 a}$ T. Dorigo, ${ }^{77 a}$ F. Gasparini, ${ }^{77 a, 77 b}$ U. Gasparini, ${ }^{77 a, 77 b}$ S. Y. Hoh, ${ }^{77 a, 77 b}$ M. Margoni, ${ }^{77 a, 77 b}$ A. T. Meneguzzo, ${ }^{77 a, 77 b}$ M. Presilla, ${ }^{77 a, 77 b}$ P. Ronchese, ${ }^{77 a, 77 b}$ R. Rossin, ${ }^{77 a, 77 b}$ G. Strong, ${ }^{77 a}$ A. Tiko, ${ }^{77 a}$ M. Tosi, ${ }^{77 a, 77 b}$ H. YARAR, ${ }^{77 a, 77 b}$ M. Zanetti, ${ }^{77 a, 77 b}$ P. Zotto, ${ }^{77 a, 77 b}$ A. Zucchetta, ${ }^{77 a, 77 b}$ G. Zumerle, ${ }^{77 a, 77 b}$ A. Braghieri, ${ }^{78 a}$ S. Calzaferri, ${ }^{78 a, 78 b}$ D. Fiorina, ${ }^{78 a, 78 b}$ P. Montagna, ${ }^{78 a, 78 b}$ S. P. Ratti, ${ }^{78 a, 78 b}$ V. Re,${ }^{78 a}$ M. Ressegotti, ${ }^{78 a, 78 b}$ C. Riccardi, ${ }^{78 a, 78 b}$ P. Salvini, ${ }^{78 a}$ I. Vai, ${ }^{78 a}$ P. Vitulo, ${ }^{78 a, 78 b}$ M. Biasini, ${ }^{79 a, 79 b}$ G. M. Bilei ${ }^{79 a}$ D. Ciangottini, ${ }^{79 a, 79 b}$ L. Fanò, ${ }^{79 a, 79 b}$ P. Lariccia, ${ }^{79 a, 79 b}$ G. Mantovani, ${ }^{79 a, 79 b}$ V. Mariani, ${ }^{79 a, 79 b}$ M. Menichelli, ${ }^{79 a}$ F. Moscatelli, ${ }^{79 a}$ A. Rossi, ${ }^{79 a, 79 b}$ A. Santocchia, ${ }^{79 a, 79 b}$ D. Spiga, ${ }^{79 a}$ T. Tedeschi, ${ }^{79 a, 79 b}$

K. Androsov, ${ }^{80 \mathrm{a}}$ P. Azzurri, ${ }^{80 \mathrm{a}}$ G. Bagliesi ${ }^{80 \mathrm{a}}$ V. Bertacchi, ${ }^{80 \mathrm{a}, 80 \mathrm{c}}$ L. Bianchini, ${ }^{80 \mathrm{a}}$ T. Boccali, ${ }^{80 \mathrm{a}} \mathrm{R}$. Castaldi, ${ }^{80 \mathrm{a}}$

M. A. Ciocci,${ }^{80 a, 80 b}$ R. Dell'Orso, ${ }^{80 a}$ M. R. Di Domenico, ${ }^{80 a, 80 b}$ S. Donato, ${ }^{80 a}$ L. Giannini,${ }^{80 a, 80 c}$ A. Giassi, ${ }^{80 a}$ M. T. Grippo, ${ }^{80 a}$

F. Ligabue ${ }^{80 a, 80 c}$ E. Manca, ${ }^{80 a, 80 c}$ G. Mandorli, ${ }^{80 a, 80 c}$ A. Messineo ${ }^{80 a, 80 b}$ F. Palla, ${ }^{80 a}$ G. Ramirez-Sanchez,${ }^{80,80 c}$

A. Rizzi, ${ }^{80 a, 80 b}$ G. Rolandi, ${ }^{80 a, 80 c}$ S. Roy Chowdhury, ${ }^{80 a, 80 c}$ A. Scribano, ${ }^{80 \mathrm{a}}$ N. Shafiei, ${ }^{80 a, 80 \mathrm{~b}}$ P. Spagnolo, ${ }^{80 \mathrm{a}}$ R. Tenchini, ${ }^{80 \mathrm{a}}$

G. Tonelli, ${ }^{80 a, 80 b}$ N. Turini, ${ }^{80 a}$ A. Venturi, ${ }^{80 a}$ P. G. Verdini, ${ }^{80 a}$ F. Cavallari, ${ }^{81 a}$ M. Cipriani, ${ }^{81 a, 81 b}$ D. Del Re,${ }^{81 a, 81 b}$

E. Di Marco, ${ }^{81 \mathrm{a}}$ M. Diemoz, ${ }^{81 \mathrm{a}}$ E. Longo, ${ }^{81 \mathrm{a}, 81 \mathrm{~b}}$ P. Meridiani, ${ }^{81 \mathrm{a}}$ G. Organtini, ${ }^{81 \mathrm{a}, 81 \mathrm{~b}}$ F. Pandolfi,,${ }^{81 \mathrm{a}}$ R. Paramatti, ${ }^{81 \mathrm{a}, 81 \mathrm{~b}}$

C. Quaranta, ${ }^{81 a, 81 b}$ S. Rahatlou, ${ }^{81 a, 81 b}$ C. Rovelli, ${ }^{81 a}$ F. Santanastasio, ${ }^{81 a, 81 b}$ L. Soffi, ${ }^{81 a, 81 b}$ R. Tramontano, ${ }^{81 a, 81 b}$ N. Amapane ${ }^{82 \mathrm{a}, 82 \mathrm{~b}}$ R. Arcidiacono, ${ }^{82 \mathrm{a}, 82 \mathrm{c}}$ S. Argiro, ${ }^{82 \mathrm{a}, 82 \mathrm{~b}}$ M. Arneodo, ${ }^{82 \mathrm{a}, 82 \mathrm{c}}$ N. Bartosik, ${ }^{82 \mathrm{a}}$ R. Bellan, ${ }^{82 a, 82 \mathrm{~b}}$ A. Bellora, ${ }^{82 a, 82 b}$ C. Biino, ${ }^{82 a}$ A. Cappati, ${ }^{82 a, 82 b}$ N. Cartiglia, ${ }^{82 a}$ S. Cometti, ${ }^{82 a}$ M. Costa, ${ }^{82 a, 82 b}$ R. Covarelli, ${ }^{82 a, 82 b}$ N. Demaria, ${ }^{82 a}$ B. Kiani, ${ }^{82 a, 82 b}$ F. Legger, ${ }^{82 a}$ C. Mariotti, ${ }^{82 a}$ S. Maselli, ${ }^{82 a}$ E. Migliore, ${ }^{82 a, 82 b}$ V. Monaco, ${ }^{82 a, 82 b}$ E. Monteil, ${ }^{82 a, 82 b}$ M. Monteno, ${ }^{82 a}$ M. M. Obertino, ${ }^{82 a, 82 b}$ G. Ortona, ${ }^{82 a}$ L. Pacher, ${ }^{82 a, 82 b}$ N. Pastrone, ${ }^{82 a}$ M. Pelliccioni, ${ }^{82 a}$ G. L. Pinna Angioni, ${ }^{82 a, 82 b}$ M. Ruspa,${ }^{82 a, 82 c}$ R. Salvatico, ${ }^{82 a, 82 b}$ F. Siviero, ${ }^{82 a, 82 b}$ V. Sola, ${ }^{82 a}$ A. Solano, ${ }^{82 a, 82 b}$ D. Soldi, ${ }^{82 a, 82 b}$ 
A. Staiano ${ }^{82 a}$ D. Trocino, ${ }^{82 a, 82 b}$ S. Belforte ${ }^{83 a}$ V. Candelise,${ }^{83 a, 83 b}$ M. Casarsa,${ }^{83 a}$ F. Cossutti, ${ }^{83 a}$ A. Da Rold,${ }^{83 a, 83 b}$ G. Della Ricca, ${ }^{83 a, 83 b}$ F. Vazzoler, ${ }^{83 a, 83 b}$ S. Dogra ${ }^{84}$ C. Huh, ${ }^{84}$ B. Kim, ${ }^{84}$ D. H. Kim,${ }^{84}$ G. N. Kim, ${ }^{84}$ J. Lee,${ }^{84}$ S. W. Lee ${ }^{84}$ C. S. Moon, ${ }^{84}$ Y. D. Oh, ${ }^{84}$ S. I. Pak,${ }^{84}$ S. Sekmen,${ }^{84}$ Y. C. Yang ${ }^{84}$ H. Kim ${ }^{85}$ D. H. Moon, ${ }^{85}$ B. Francois, ${ }^{86}$ T. J. Kim, ${ }^{86}$ J. Park, ${ }^{86}$ S. Cho, ${ }^{87}$ S. Choi, ${ }^{87}$ Y. Go, ${ }^{87}$ S. Ha, ${ }^{87}$ B. Hong, ${ }^{87}$ K. Lee, ${ }^{87}$ K. S. Lee,${ }^{87}$ J. Lim, ${ }^{87}$ J. Park, ${ }^{87}$ S. K. Park,,${ }^{87}$ J. Yoo, ${ }^{87}$ J. Goh ${ }^{88}$ A. Gurtu, ${ }^{88}$ H. S. Kim, ${ }^{89}$ Y. Kim,${ }^{89}$ J. Almond, ${ }^{90}$ J. H. Bhyun, ${ }^{90}$ J. Choi,,${ }^{90}$ S. Jeon, ${ }^{90}$ J. Kim, ${ }^{90}$ J. S. Kim, ${ }^{90}$ S. Ko, ${ }^{90}$ H. Kwon, ${ }^{90}$ H. Lee, ${ }^{90}$ K. Lee, ${ }^{90}$ S. Lee,${ }^{90}$ K. Nam, ${ }^{90}$ B. H. Oh, ${ }^{90}$ M. Oh, ${ }^{90}$ S. B. Oh ${ }^{90}$ B. C. Radburn-Smith, ${ }^{90}$ H. Seo,${ }^{90}$ U.K. Yang, ${ }^{90}$ I. Yoon,${ }^{90}$ D. Jeon, ${ }^{91}$ J. H. Kim,${ }^{91}$ B. Ko, ${ }^{91}$ J. S. H. Lee, ${ }^{91}$ I. C. Park,${ }^{91}$ Y. Roh, ${ }^{91}$ D. Song, ${ }^{91}$ I. J. Watson, ${ }^{91}$ H. D. Yoo, ${ }^{92}$ Y. Choi, ${ }^{93}$ C. Hwang, ${ }^{93}$ Y. Jeong, ${ }^{93}$ H. Lee, ${ }^{93}$ J. Lee, ${ }^{93}$ Y. Lee,,${ }^{93}$ I. Yu,${ }^{93}$ V. Veckalns, ${ }^{94, p p}$ A. Juodagalvis, ${ }^{95}$ A. Rinkevicius, ${ }^{95}$ G. Tamulaitis, ${ }^{95}$ W. A. T. Wan Abdullah, ${ }^{96}$ M. N. Yusli, ${ }^{96}$ Z. Zolkapli, ${ }^{96}$ J. F. Benitez, ${ }^{97}$

A. Castaneda Hernandez, ${ }^{97}$ J. A. Murillo Quijada, ${ }^{97}$ L. Valencia Palomo, ${ }^{97}$ H. Castilla-Valdez, ${ }^{98}$ E. De La Cruz-Burelo, ${ }^{98}$ I. Heredia-De La Cruz, ${ }^{98,4 q}$ R. Lopez-Fernandez, ${ }^{98}$ A. Sanchez-Hernandez, ${ }^{98}$ S. Carrillo Moreno, ${ }^{99}$ C. Oropeza Barrera, ${ }^{99}$ M. Ramirez-Garcia, ${ }^{99}$ F. Vazquez Valencia,${ }^{99}$ J. Eysermans, ${ }^{100}$ I. Pedraza ${ }^{100}$ H. A. Salazar Ibarguen, ${ }^{100}$ C. Uribe Estrada, ${ }^{100}$ A. Morelos Pineda, ${ }^{101}$ J. Mijuskovic, ${ }^{102, e}$ N. Raicevic, ${ }^{102}$ D. Krofcheck, ${ }^{103}$ S. Bheesette, ${ }^{104}$ P. H. Butler, ${ }^{104}$ A. Ahmad, ${ }^{105}$ M. I. Asghar, ${ }^{105}$ M. I. M. Awan, ${ }^{105}$ Q. Hassan, ${ }^{105}$ H. R. Hoorani, ${ }^{105}$ W. A. Khan, ${ }^{105}$ M. A. Shah,${ }^{105}$ M. Shoaib,${ }^{105}$ M. Waqas, ${ }^{105}$ V. Avati, ${ }^{106}$ L. Grzanka, ${ }^{106}$ M. Malawski, ${ }^{106}$ H. Bialkowska, ${ }^{107}$ M. Bluj, ${ }^{107}$ B. Boimska, ${ }^{107}$ T. Frueboes, ${ }^{107}$ M. Górski, ${ }^{107}$ M. Kazana, ${ }^{107}$ M. Szleper, ${ }^{107}$ P. Traczyk, ${ }^{107}$ P. Zalewski, ${ }^{107}$ K. Bunkowski, ${ }^{108}$ A. Byszuk, ${ }^{108, \text { rr }}$ K. Doroba, ${ }^{108}$ A. Kalinowski, ${ }^{108}$ M. Konecki, ${ }^{108}$ J. Krolikowski, ${ }^{108}$ M. Olszewski, ${ }^{108}$ M. Walczak, ${ }^{108}$ M. Araujo, ${ }^{109}$ P. Bargassa, ${ }^{109}$ D. Bastos, ${ }^{109}$ A. Di Francesco, ${ }^{109}$ P. Faccioli, ${ }^{109}$ B. Galinhas, ${ }^{109}$ M. Gallinaro, ${ }^{109}$ J. Hollar, ${ }^{109}$ N. Leonardo, ${ }^{109}$ T. Niknejad, ${ }^{109}$ J. Seixas, ${ }^{109}$ K. Shchelina, ${ }^{109}$ O. Toldaiev, ${ }^{109}$ J. Varela, ${ }^{109}$ S. Afanasiev, ${ }^{110}$ P. Bunin, ${ }^{110}$ M. Gavrilenko, ${ }^{110}$ I. Golutvin, ${ }^{110}$ I. Gorbunov, ${ }^{110}$ A. Kamenev, ${ }^{110}$ V. Karjavine, ${ }^{110}$ A. Lanev, ${ }^{110}$ A. Malakhov,${ }^{110}$ V. Matveev, ${ }^{110, s s, t t}$ P. Moisenz, ${ }^{110}$ V. Palichik, ${ }^{110}$ V. Perelygin, ${ }^{110}$ M. Savina, ${ }^{110}$ D. Seitova, ${ }^{110}$ V. Shalaev, ${ }^{110}$ S. Shmatov, ${ }^{110}$ S. Shulha, ${ }^{110}$ V. Smirnov,${ }^{110}$ O. Teryaev, ${ }^{110}$ N. Voytishin, ${ }^{110}$ A. Zarubin, ${ }^{110}$ I. Zhizhin, ${ }^{110}$ G. Gavrilov, ${ }^{111}$ V. Golovtcov, ${ }^{111}$ Y. Ivanov, ${ }^{111}$ V. Kim, ${ }^{111, \text { uu }}$ E. Kuznetsova, ${ }^{111, v v}$ V. Murzin, ${ }^{111}$ V. Oreshkin, ${ }^{111}$ I. Smirnov,${ }^{111}$ D. Sosnov,${ }^{111}$ V. Sulimov, ${ }^{111}$ L. Uvarov, ${ }^{111}$ S. Volkov, ${ }^{111}$ A. Vorobyev, ${ }^{111}$ Yu. Andreev, ${ }^{112}$ A. Dermenev, ${ }^{112}$ S. Gninenko, ${ }^{112}$ N. Golubev, ${ }^{112}$ A. Karneyeu, ${ }^{112}$ M. Kirsanov, ${ }^{112}$

N. Krasnikov, ${ }^{112}$ A. Pashenkov,${ }^{112}$ G. Pivovarov, ${ }^{112}$ D. Tlisov, ${ }^{112}$ A. Toropin, ${ }^{112}$ V. Epshteyn, ${ }^{113}$ V. Gavrilov, ${ }^{113}$ N. Lychkovskaya, ${ }^{113}$ A. Nikitenko, ${ }^{113, w w}$ V. Popov, ${ }^{113}$ I. Pozdnyakov, ${ }^{113}$ G. Safronov, ${ }^{113}$ A. Spiridonov, ${ }^{113}$ A. Stepennov, ${ }^{113}$ M. Toms, ${ }^{113}$ E. Vlasov, ${ }^{113}$ A. Zhokin, ${ }^{113}$ T. Aushev, ${ }^{114}$ R. Chistov, ${ }^{115 x x}$ M. Danilov, ${ }^{115, x x}$ A. Oskin, ${ }^{115}$ P. Parygin, ${ }^{115}$ S. Polikarpov, ${ }^{115, \mathrm{xx}}$ V. Andreev, ${ }^{116}$ M. Azarkin, ${ }^{116}$ I. Dremin, ${ }^{116}$ M. Kirakosyan, ${ }^{116}$ A. Terkulov, ${ }^{116}$ A. Belyaev,${ }^{117}$ E. Boos, ${ }^{117}$ M. Dubinin, ${ }^{117, y y}$ L. Dudko, ${ }^{117}$ A. Ershov, ${ }^{117}$ A. Gribushin, ${ }^{117}$ V. Klyukhin, ${ }^{117}$ O. Kodolova, ${ }^{117}$ I. Lokhtin, ${ }^{117}$

S. Obraztsov, ${ }^{117}$ S. Petrushanko, ${ }^{117}$ V. Savrin, ${ }^{117}$ A. Snigirev, ${ }^{117}$ V. Blinov, ${ }^{118, z z}$ T. Dimova, ${ }^{118, z z}$ L. Kardapoltsev, ${ }^{118, z z}$ I. Ovtin, ${ }^{18, z z}$ Y. Skovpen, ${ }^{118, z z}$ I. Azhgirey, ${ }^{119}$ I. Bayshev, ${ }^{119}$ V. Kachanov,${ }^{119}$ A. Kalinin, ${ }^{119}$ D. Konstantinov, ${ }^{119}$ V. Petrov, ${ }^{119}$ R. Ryutin, ${ }^{119}$ A. Sobol, ${ }^{119}$ S. Troshin, ${ }^{119}$ N. Tyurin, ${ }^{119}$ A. Uzunian, ${ }^{119}$ A. Volkov, ${ }^{119}$ A. Babaev, ${ }^{120}$ A. Iuzhakov, ${ }^{120}$ V. Okhotnikov, ${ }^{120}$ L. Sukhikh, ${ }^{120}$ V. Borchsh, ${ }^{121}$ V. Ivanchenko, ${ }^{121}$ E. Tcherniaev, ${ }^{121}$ P. Adzic, ${ }^{122, \text { aaa }}$ P. Cirkovic, ${ }^{122}$ M. Dordevic, ${ }^{122}$ P. Milenovic, ${ }^{122}$ J. Milosevic, ${ }^{122}$ M. Aguilar-Benitez, ${ }^{123}$ J. Alcaraz Maestre, ${ }^{123}$ A. Álvarez Fernández, ${ }^{123}$ I. Bachiller, ${ }^{123}$ M. Barrio Luna, ${ }^{123}$ Cristina F. Bedoya, ${ }^{123}$ J. A. Brochero Cifuentes, ${ }^{123}$ C. A. Carrillo Montoya, ${ }^{123}$ M. Cepeda ${ }^{123}$ M. Cerrada, ${ }^{123}$ N. Colino, ${ }^{123}$ B. De La Cruz, ${ }^{123}$ A. Delgado Peris, ${ }^{123}$ J. P. Fernández Ramos, ${ }^{123}$ J. Flix, ${ }^{123}$ M. C. Fouz, ${ }^{123}$ O. Gonzalez Lopez, ${ }^{123}$ S. Goy Lopez, ${ }^{123}$ J. M. Hernandez, ${ }^{123}$ M. I. Josa, ${ }^{123}$ D. Moran, ${ }^{123}$ Á. Navarro Tobar, ${ }^{123}$ A. Pérez-Calero Yzquierdo, ${ }^{123}$ J. Puerta Pelayo, ${ }^{123}$ I. Redondo, ${ }^{123}$ L. Romero, ${ }^{123}$ S. Sánchez Navas, ${ }^{123}$ M. S. Soares, ${ }^{123}$ A. Triossi, ${ }^{123}$ C. Willmott, ${ }^{123}$ C. Albajar, ${ }^{124}$ J. F. de Trocóniz, ${ }^{124}$ R. Reyes-Almanza, ${ }^{124}$ B. Alvarez Gonzalez, ${ }^{125}$ J. Cuevas, ${ }^{125}$ C. Erice ${ }^{125}$ J. Fernandez Menendez, ${ }^{125}$ S. Folgueras, ${ }^{125}$ I. Gonzalez Caballero, ${ }^{125}$ E. Palencia Cortezon, ${ }^{125}$ C. Ramón Álvarez, ${ }^{125}$ V. Rodríguez Bouza, ${ }^{125}$ S. Sanchez Cruz, ${ }^{125}$ A. Trapote, ${ }^{125}$ I. J. Cabrillo, ${ }^{126}$ A. Calderon, ${ }^{126}$ B. Chazin Quero, ${ }^{126}$ J. Duarte Campderros, ${ }^{126}$ M. Fernandez, ${ }^{126}$ P. J. Fernández Manteca, ${ }^{126}$ A. García Alonso, ${ }^{126}$ G. Gomez, ${ }^{126}$ C. Martinez Rivero, ${ }^{126}$ P. Martinez Ruiz del Arbol,${ }^{126}$ F. Matorras, ${ }^{126}$ J. Piedra Gomez, ${ }^{126}$ C. Prieels, ${ }^{126}$ F. Ricci-Tam, ${ }^{126}$ T. Rodrigo, ${ }^{126}$ A. Ruiz-Jimeno, ${ }^{126}$ L. Russo, ${ }^{126, b b b}$ L. Scodellaro, ${ }^{126}$ I. Vila, ${ }^{126}$ J. M. Vizan Garcia, ${ }^{126}$ MK Jayananda ${ }^{127}$ B. Kailasapathy, ${ }^{127, c c c}$ D. U. J. Sonnadara, ${ }^{127}$ DDC Wickramarathna, ${ }^{127}$ W. G. D. Dharmaratna, ${ }^{128}$

K. Liyanage,${ }^{128}$ N. Perera, ${ }^{128}$ N. Wickramage, ${ }^{128}$ T. K. Aarrestad, ${ }^{129}$ D. Abbaneo, ${ }^{129}$ B. Akgun, ${ }^{129}$ E. Auffray, ${ }^{129}$ G. Auzinger, ${ }^{129}$ J. Baechler, ${ }^{129}$ P. Baillon, ${ }^{129}$ A. H. Ball, ${ }^{129}$ D. Barney, ${ }^{129}$ J. Bendavid, ${ }^{129}$ N. Beni,${ }^{129}$ M. Bianco, ${ }^{129}$ A. Bocci, ${ }^{129}$ P. Bortignon, ${ }^{129}$ E. Bossini, ${ }^{129}$ E. Brondolin, ${ }^{129}$ T. Camporesi, ${ }^{129}$ G. Cerminara, ${ }^{129}$ L. Cristella, ${ }^{129}$ D. d'Enterria, ${ }^{129}$ A. Dabrowski, ${ }^{129}$ N. Daci, ${ }^{129}$ V. Daponte, ${ }^{129}$ A. David, ${ }^{129}$ A. De Roeck, ${ }^{129}$ M. Deile, ${ }^{129}$ R. Di Maria, ${ }^{129}$ 
M. Dobson, ${ }^{129}$ M. Dünser, ${ }^{129}$ N. Dupont, ${ }^{129}$ A. Elliott-Peisert, ${ }^{129}$ N. Emriskova, ${ }^{129}$ F. Fallavollita, ${ }^{129, d d d}$ D. Fasanella, ${ }^{129}$ S. Fiorendi, ${ }^{129}$ G. Franzoni, ${ }^{129}$ J. Fulcher, ${ }^{129}$ W. Funk, ${ }^{129}$ S. Giani,${ }^{129}$ D. Gigi,${ }^{129}$ K. Gill, ${ }^{129}$ F. Glege, ${ }^{129}$ L. Gouskos, ${ }^{129}$ M. Guilbaud, ${ }^{129}$ D. Gulhan, ${ }^{129}$ M. Haranko, ${ }^{129}$ J. Hegeman, ${ }^{129}$ Y. Iiyama, ${ }^{129}$ V. Innocente, ${ }^{129}$ T. James, ${ }^{129}$ P. Janot,${ }^{129}$ J. Kaspar, ${ }^{129}$ J. Kieseler, ${ }^{129}$ M. Komm, ${ }^{129}$ N. Kratochwil, ${ }^{129}$ C. Lange, ${ }^{129}$ P. Lecoq, ${ }^{129}$ K. Long, ${ }^{129}$ C. Lourenço, ${ }^{129}$ L. Malgeri, ${ }^{129}$ M. Mannelli, ${ }^{129}$ A. Massironi, ${ }^{129}$ F. Meijers, ${ }^{129}$ S. Mersi, ${ }^{129}$ E. Meschi, ${ }^{129}$ F. Moortgat,${ }^{129}$ M. Mulders, ${ }^{129}$ J. Ngadiuba, ${ }^{129}$ J. Niedziela, ${ }^{129}$ S. Orfanelli, ${ }^{129}$ L. Orsini, ${ }^{129}$ F. Pantaleo, ${ }^{129, t}$ L. Pape, ${ }^{129}$ E. Perez, ${ }^{129}$ M. Peruzzi, ${ }^{129}$ A. Petrilli, ${ }^{129}$ G. Petrucciani, ${ }^{129}$ A. Pfeiffer, ${ }^{129}$ M. Pierini, ${ }^{129}$ D. Rabady, ${ }^{129}$ A. Racz, ${ }^{129}$ M. Rieger, ${ }^{129}$ M. Rovere, ${ }^{129}$ H. Sakulin, ${ }^{129}$ J. Salfeld-Nebgen, ${ }^{129}$ S. Scarfi, ${ }^{129}$ C. Schäfer, ${ }^{129}$ C. Schwick, ${ }^{129}$ M. Selvaggi, ${ }^{129}$ A. Sharma, ${ }^{129}$ P. Silva, ${ }^{129}$ W. Snoeys, ${ }^{129}$ P. Sphicas, ${ }^{129, \text { eee }}$ J. Steggemann, ${ }^{129}$ S. Summers, ${ }^{129}$ V. R. Tavolaro, ${ }^{129}$ D. Treille, ${ }^{129}$ A. Tsirou, ${ }^{129}$

G. P. Van Onsem, ${ }^{129}$ A. Vartak, ${ }^{129}$ M. Verzetti, ${ }^{129}$ K. A. Wozniak, ${ }^{129}$ W. D. Zeuner, ${ }^{129}$ L. Caminada, ${ }^{130, f f}$ W. Erdmann, ${ }^{130}$ R. Horisberger, ${ }^{130}$ Q. Ingram, ${ }^{130}$ H. C. Kaestli, ${ }^{130}$ D. Kotlinski, ${ }^{130}$ U. Langenegger, ${ }^{130}$ T. Rohe, ${ }^{130}$ M. Backhaus, ${ }^{131}$ P. Berger, ${ }^{131}$ A. Calandri, ${ }^{131}$ N. Chernyavskaya, ${ }^{131}$ G. Dissertori, ${ }^{131}$ M. Dittmar,${ }^{131}$ M. Donegà, ${ }^{131}$ C. Dorfer, ${ }^{131}$ T. Gadek, ${ }^{131}$ T. A. Gómez Espinosa, ${ }^{131}$ C. Grab, ${ }^{131}$ D. Hits, ${ }^{131}$ W. Lustermann, ${ }^{131}$ A.-M. Lyon, ${ }^{131}$ R. A. Manzoni, ${ }^{131}$ M. T. Meinhard, ${ }^{131}$ F. Micheli, ${ }^{131}$ P. Musella, ${ }^{131}$ F. Nessi-Tedaldi, ${ }^{131}$ F. Pauss,${ }^{131}$ V. Perovic, ${ }^{131}$ G. Perrin, ${ }^{131}$ L. Perrozzi, ${ }^{131}$ S. Pigazzini, ${ }^{131}$ M. G. Ratti, ${ }^{131}$ M. Reichmann, ${ }^{131}$ C. Reissel, ${ }^{131}$ T. Reitenspiess, ${ }^{131}$ B. Ristic, ${ }^{131}$ D. Ruini, ${ }^{131}$ D. A. Sanz Becerra, ${ }^{131}$

M. Schönenberger ${ }^{131}$ L. Shchutska, ${ }^{131}$ V. Stampf,${ }^{131}$ M. L. Vesterbacka Olsson, ${ }^{131}$ R. Wallny, ${ }^{131}$ D. H. Zhu, ${ }^{131}$ C. Amsler, ${ }^{132, g g g}$ C. Botta, ${ }^{132}$ D. Brzhechko, ${ }^{132}$ M. F. Canelli, ${ }^{132}$ A. De Cosa,${ }^{132}$ R. Del Burgo, ${ }^{132}$ J. K. Heikkilä, ${ }^{132}$ M. Huwiler, ${ }^{132}$ A. Jofrehei, ${ }^{132}$ B. Kilminster, ${ }^{132}$ S. Leontsinis, ${ }^{132}$ A. Macchiolo, ${ }^{132}$ P. Meiring, ${ }^{132}$ V. M. Mikuni, ${ }^{132}$ U. Molinatti, ${ }^{132}$ I. Neutelings, ${ }^{132}$ G. Rauco, ${ }^{132}$ A. Reimers, ${ }^{132}$ P. Robmann, ${ }^{132}$ K. Schweiger, ${ }^{132}$ Y. Takahashi,${ }^{132}$ S. Wertz, ${ }^{132}$ C. Adloff, ${ }^{133, \text { hhh }}$ C. M. Kuo, ${ }^{133}$ W. Lin, ${ }^{133}$ A. Roy, ${ }^{133}$ T. Sarkar, ${ }^{133, \text { ii }}$ S. S. Yu, ${ }^{133}$ L. Ceard,${ }^{134}$ P. Chang, ${ }^{134}$ Y. Chao, ${ }^{134}$ K. F. Chen, ${ }^{134}$ P. H. Chen, ${ }^{134}$ W.-S. Hou, ${ }^{134}$ Y. y. Li, ${ }^{134}$ R.-S. Lu, ${ }^{134}$ E. Paganis, ${ }^{134}$ A. Psallidas, ${ }^{134}$ A. Steen, ${ }^{134}$ E. Yazgan, ${ }^{134}$ B. Asavapibhop, ${ }^{135}$ C. Asawatangtrakuldee, ${ }^{135}$ N. Srimanobhas, ${ }^{135}$ F. Boran, ${ }^{136}$ S. Damarseckin, ${ }^{136, i i i}$ Z. S. Demiroglu, ${ }^{136}$ F. Dolek, ${ }^{136}$ C. Dozen, ${ }^{136, j j j}$ I. Dumanoglu,${ }^{136, k k k}$ E. Eskut, ${ }^{136}$ G. Gokbulut, ${ }^{136}$ Y. Guler, ${ }^{136}$ E. Gurpinar Guler, ${ }^{136,111}$ I. Hos, ${ }^{136, m m m}$ C. Isik, ${ }^{136}$ E. E. Kangal, ${ }^{136, n n n}$ O. Kara, ${ }^{136}$ A. Kayis Topaksu, ${ }^{136}$ U. Kiminsu, ${ }^{136}$ G. Onengut, ${ }^{136}$ K. Ozdemir, ${ }^{136,000}$ A. Polatoz, ${ }^{136}$ A. E. Simsek, ${ }^{136}$ B. Tali, ${ }^{136, p p p}$ U. G. Tok, ${ }^{136}$ S. Turkcapar, ${ }^{136}$ I. S. Zorbakir, ${ }^{136}$ C. Zorbilmez, ${ }^{136}$ B. Isildak, ${ }^{137, \text { qqq }}$ G. Karapinar, ${ }^{177, \text { rrr }}$ K. Ocalan, ${ }^{137, \text { sss }}$ M. Yalvac, ${ }^{137, \text { tt }}$ I. O. Atakisi, ${ }^{138}$ E. Gülmez, ${ }^{138}$ M. Kaya, ${ }^{138, \text { uuu }}$ O. Kaya, ${ }^{138, v v v}$ Ö. Özçelik, ${ }^{138}$ S. Tekten, ${ }^{138, \text { www }}$ E. A. Yetkin, ${ }^{138, \text { xxx }}$ A. Cakir, ${ }^{139}$ K. Cankocak, ${ }^{139, k k k}$ Y. Komurcu, ${ }^{139}$ S. Sen, ${ }^{139, y y y}$ F. Aydogmus Sen, ${ }^{140}$ S. Cerci, ${ }^{140, p p p}$ B. Kaynak, ${ }^{140}$ S. Ozkorucuklu, ${ }^{140}$ D. Sunar Cerci, ${ }^{140, p p p}$ B. Grynyov, ${ }^{141}$ L. Levchuk,${ }^{142}$ E. Bhal,${ }^{143}$ S. Bologna,${ }^{143}$ J. J. Brooke, ${ }^{143}$ D. Burns, ${ }^{143, z z z}$ E. Clement, ${ }^{143}$ D. Cussans, ${ }^{143}$ H. Flacher, ${ }^{143}$ J. Goldstein, ${ }^{143}$ G. P. Heath,${ }^{143}$ H. F. Heath, ${ }^{143}$ L. Kreczko, ${ }^{143}$ B. Krikler, ${ }^{143}$ S. Paramesvaran, ${ }^{143}$ T. Sakuma, ${ }^{143}$ S. Seif El Nasr-Storey, ${ }^{143}$ V. J. Smith, ${ }^{143}$ J. Taylor, ${ }^{143}$ A. Titterton, ${ }^{143}$ K. W. Bell, ${ }^{144}$ A. Belyaev, ${ }^{144, \text { aaaa }}$ C. Brew, ${ }^{144}$ R. M. Brown, ${ }^{144}$ D. J. A. Cockerill, ${ }^{144}$ K. V. Ellis, ${ }^{144}$ K. Harder, ${ }^{144}$ S. Harper,${ }^{144}$ J. Linacre, ${ }^{144}$ K. Manolopoulos, ${ }^{144}$ D. M. Newbold, ${ }^{144}$ E. Olaiya, ${ }^{144}$ D. Petyt, ${ }^{144}$ T. Reis,${ }^{144}$ T. Schuh, ${ }^{144}$ C. H. Shepherd-Themistocleous, ${ }^{144}$ A. Thea,${ }^{144}$ I. R. Tomalin, ${ }^{144}$ T. Williams, ${ }^{144}$ R. Bainbridge, ${ }^{145}$ P. Bloch, ${ }^{145}$ S. Bonomally, ${ }^{145}$ J. Borg, ${ }^{145}$ S. Breeze,${ }^{145}$ O. Buchmuller, ${ }^{145}$ A. Bundock, ${ }^{145}$ V. Cepaitis, ${ }^{145}$ G. S. Chahal, ${ }^{145, \text { bbbb }}$ D. Colling, ${ }^{145}$ P. Dauncey, ${ }^{145}$ G. Davies, ${ }^{145}$ M. Della Negra, ${ }^{145}$ P. Everaerts, ${ }^{145}$ G. Fedi, ${ }^{145}$ G. Hall, ${ }^{145}$ G. Iles, ${ }^{145}$ J. Langford,${ }^{145}$ L. Lyons, ${ }^{145}$ A.-M. Magnan, ${ }^{145}$ S. Malik, ${ }^{145}$ A. Martelli, ${ }^{145}$ V. Milosevic, ${ }^{145}$ J. Nash, ${ }^{145, \mathrm{cccc}}$ V. Palladino, ${ }^{145}$ M. Pesaresi,${ }^{145}$ D. M. Raymond ${ }^{145}$ A. Richards, ${ }^{145}$ A. Rose,${ }^{145}$ E. Scott, ${ }^{145}$ C. Seez ${ }^{145}$ A. Shtipliyski, ${ }^{145}$ M. Stoye,${ }^{145}$ A. Tapper, ${ }^{145}$ K. Uchida, ${ }^{145}$ T. Virdee,${ }^{145, t}$ N. Wardle, ${ }^{145}$ S. N. Webb,${ }^{145}$ D. Winterbottom, ${ }^{145}$ A. G. Zecchinelli, ${ }^{145}$ S. C. Zenz, ${ }^{145}$ J. E. Cole,${ }^{146}$ P. R. Hobson, ${ }^{146}$ A. Khan, ${ }^{146}$ P. Kyberd, ${ }^{146}$ C. K. Mackay, ${ }^{146}$ I. D. Reid, ${ }^{146}$ L. Teodorescu, ${ }^{146}$ S. Zahid, ${ }^{146}$ A. Brinkerhoff,${ }^{147}$ K. Call, ${ }^{147}$ B. Caraway, ${ }^{147}$ J. Dittmann, ${ }^{147}$ K. Hatakeyama, ${ }^{147}$ A. R. Kanuganti, ${ }^{147}$ C. Madrid, ${ }^{147}$ B. McMaster, ${ }^{147}$ N. Pastika, ${ }^{147}$ S. Sawant, ${ }^{147}$ C. Smith, ${ }^{147}$ R. Bartek, ${ }^{148}$ A. Dominguez, ${ }^{148}$ R. Uniyal, ${ }^{148}$ A. M. Vargas Hernandez, ${ }^{148}$ A. Buccilli, ${ }^{149}$ O. Charaf, ${ }^{149}$ S. I. Cooper, ${ }^{149}$ S. V. Gleyzer, ${ }^{149}$ C. Henderson, ${ }^{149}$ P. Rumerio, ${ }^{149}$ C. West, ${ }^{149}$ A. Akpinar, ${ }^{150}$ A. Albert, ${ }^{150}$ D. Arcaro, ${ }^{150}$ C. Cosby, ${ }^{150}$ Z. Demiragli, ${ }^{150}$ D. Gastler, ${ }^{150}$ C. Richardson, ${ }^{150}$ J. Rohlf,${ }^{150}$ K. Salyer, ${ }^{150}$ D. Sperka, ${ }^{150}$ D. Spitzbart, ${ }^{150}$ I. Suarez, ${ }^{150}$ S. Yuan, ${ }^{150}$ D. Zou, ${ }^{150}$ G. Benelli, ${ }^{151}$ B. Burkle, ${ }^{151}$ X. Coubez, ${ }^{151, u}$ D. Cutts, ${ }^{151}$ Y. t. Duh, ${ }^{151}$ M. Hadley, ${ }^{151}$ U. Heintz, ${ }^{151}$ J. M. Hogan, ${ }^{151, \text { dddd }}$ K. H. M. Kwok, ${ }^{151}$ E. Laird, ${ }^{151}$ G. Landsberg ${ }^{151}$ K. T. Lau, ${ }^{151}$ J. Lee, ${ }^{151}$ M. Narain, ${ }^{151}$ S. Sagir, ${ }^{151, e e e}$ R. Syarif, ${ }^{151}$ E. Usai, ${ }^{151}$ W. Y. Wong, ${ }^{151}$ D. Yu, ${ }^{151}$ W. Zhang, ${ }^{151}$ R. Band,${ }^{152}$ C. Brainerd ${ }^{152}$ R. Breedon, ${ }^{152}$

M. Calderon De La Barca Sanchez, ${ }^{152}$ M. Chertok, ${ }^{152}$ J. Conway, ${ }^{152}$ R. Conway, ${ }^{152}$ P. T. Cox, ${ }^{152}$ R. Erbacher, ${ }^{152}$ C. Flores, ${ }^{152}$ G. Funk, ${ }^{152}$ F. Jensen, ${ }^{152}$ W. Ko, ${ }^{152, a}$ O. Kukral, ${ }^{152}$ R. Lander, ${ }^{152}$ M. Mulhearn, ${ }^{152}$ D. Pellett, ${ }^{152}$ J. Pilot, ${ }^{152}$ M. Shi, ${ }^{152}$ 
D. Taylor,${ }^{152}$ K. Tos, ${ }^{152}$ M. Tripathi, ${ }^{152}$ Y. Yao, ${ }^{152}$ F. Zhang, ${ }^{152}$ M. Bachtis, ${ }^{153}$ C. Bravo, ${ }^{153}$ R. Cousins, ${ }^{153}$ A. Dasgupta, ${ }^{153}$ A. Florent, ${ }^{153}$ D. Hamilton, ${ }^{153}$ J. Hauser, ${ }^{153}$ M. Ignatenko, ${ }^{153}$ T. Lam, ${ }^{153}$ N. Mccoll, ${ }^{153}$ W. A. Nash, ${ }^{153}$ S. Regnard, ${ }^{153}$ D. Saltzberg, ${ }^{153}$ C. Schnaible, ${ }^{153}$ B. Stone, ${ }^{153}$ V. Valuev, ${ }^{153}$ K. Burt, ${ }^{154}$ Y. Chen, ${ }^{154}$ R. Clare, ${ }^{154}$ J. W. Gary, ${ }^{154}$ S. M. A. Ghiasi Shirazi, ${ }^{154}$ G. Hanson, ${ }^{154}$ G. Karapostoli, ${ }^{154}$ O. R. Long,${ }^{154}$ N. Manganelli, ${ }^{154}$ M. Olmedo Negrete, ${ }^{154}$ M. I. Paneva, ${ }^{154}$ W. Si, ${ }^{154}$ S. Wimpenny, ${ }^{154}$ Y. Zhang, ${ }^{154}$ J. G. Branson, ${ }^{155}$ P. Chang, ${ }^{155}$ S. Cittolin, ${ }^{155}$ S. Cooperstein, ${ }^{155}$ N. Deelen, ${ }^{155}$ M. Derdzinski, ${ }^{155}$ J. Duarte, ${ }^{155}$ R. Gerosa, ${ }^{155}$ D. Gilbert, ${ }^{155}$ B. Hashemi, ${ }^{155}$ D. Klein,${ }^{155}$ V. Krutelyov, ${ }^{155}$ J. Letts, ${ }^{155}$ M. Masciovecchio, ${ }^{155}$ S. May, ${ }^{155}$ S. Padhi,${ }^{155}$ M. Pieri, ${ }^{155}$ V. Sharma, ${ }^{155}$ M. Tadel,${ }^{155}$ F. Würthwein, ${ }^{155}$ A. Yagil ${ }^{155}$ N. Amin, ${ }^{156}$ R. Bhandari, ${ }^{156}$ C. Campagnari, ${ }^{156}$ M. Citron, ${ }^{156}$ A. Dorsett, ${ }^{156}$ V. Dutta, ${ }^{156}$ J. Incandela, ${ }^{156}$ B. Marsh, ${ }^{156}$ H. Mei, ${ }^{156}$ A. Ovcharova, ${ }^{156}$ H. Qu, ${ }^{156}$ M. Quinnan, ${ }^{156}$ J. Richman, ${ }^{156}$ U. Sarica, ${ }^{156}$ D. Stuart, ${ }^{156}$ S. Wang, ${ }^{156}$ D. Anderson, ${ }^{157}$ A. Bornheim, ${ }^{157}$ O. Cerri, ${ }^{157}$ I. Dutta, ${ }^{157}$ J. M. Lawhorn, ${ }^{157}$ N. Lu, ${ }^{157}$ J. Mao, ${ }^{157}$ H. B. Newman, ${ }^{157}$

T. Q. Nguyen, ${ }^{157}$ J. Pata, ${ }^{157}$ M. Spiropulu, ${ }^{157}$ J. R. Vlimant, ${ }^{157}$ S. Xie, ${ }^{157}$ Z. Zhang, ${ }^{157}$ R. Y. Zhu, ${ }^{157}$ J. Alison, ${ }^{158}$

M. B. Andrews, ${ }^{158}$ T. Ferguson, ${ }^{158}$ T. Mudholkar, ${ }^{158}$ M. Paulini, ${ }^{158}$ M. Sun, ${ }^{158}$ I. Vorobiev ${ }^{158}$ M. Weinberg, ${ }^{158}$ J. P. Cumalat, ${ }^{159}$ W. T. Ford, ${ }^{159}$ E. MacDonald, ${ }^{159}$ T. Mulholland, ${ }^{159}$ R. Patel, ${ }^{159}$ A. Perloff, ${ }^{159}$ K. Stenson, ${ }^{159}$ K. A. Ulmer, ${ }^{159}$ S. R. Wagner, ${ }^{159}$ J. Alexander, ${ }^{160}$ Y. Cheng, ${ }^{160}$ J. Chu, ${ }^{160}$ D. J. Cranshaw, ${ }^{160}$ A. Datta, ${ }^{160}$ A. Frankenthal, ${ }^{160}$ K. Mcdermott, ${ }^{160}$ J. Monroy, ${ }^{160}$ J. R. Patterson, ${ }^{160}$ D. Quach, ${ }^{160}$ A. Ryd ${ }^{160}$ W. Sun, ${ }^{160}$ S. M. Tan, ${ }^{160}$ Z. Tao, ${ }^{160}$ J. Thom, ${ }^{160}$ P. Wittich, ${ }^{160}$ M. Zientek, ${ }^{160}$ S. Abdullin, ${ }^{161}$ M. Albrow ${ }^{161}$ M. Alyari, ${ }^{161}$ G. Apollinari, ${ }^{161}$ A. Apresyan,${ }^{161}$ A. Apyan, ${ }^{161}$ S. Banerjee, ${ }^{161}$ L. A. T. Bauerdick, ${ }^{161}$ A. Beretvas, ${ }^{161}$ D. Berry, ${ }^{161}$ J. Berryhill, ${ }^{161}$ P. C. Bhat, ${ }^{161}$ K. Burkett,${ }^{161}$ J. N. Butler, ${ }^{161}$ A. Canepa, ${ }^{161}$ G. B. Cerati, ${ }^{161}$ H. W. K. Cheung, ${ }^{161}$ F. Chlebana, ${ }^{161}$ M. Cremonesi, ${ }^{161}$ K. F. Di Petrillo, ${ }^{161}$ V. D. Elvira, ${ }^{161}$ J. Freeman, ${ }^{161}$ Z. Gecse, ${ }^{161}$ E. Gottschalk, ${ }^{161}$ L. Gray, ${ }^{161}$ D. Green, ${ }^{161}$ S. Grünendahl, ${ }^{161}$ O. Gutsche, ${ }^{161}$ R. M. Harris, ${ }^{161}$ S. Hasegawa, ${ }^{161}$ R. Heller, ${ }^{161}$ T. C. Herwig, ${ }^{161}$ J. Hirschauer, ${ }^{161}$ B. Jayatilaka, ${ }^{161}$ S. Jindariani, ${ }^{161}$ M. Johnson, ${ }^{161}$ U. Joshi, ${ }^{161}$ T. Klijnsma, ${ }^{161}$ B. Klima, ${ }^{161}$ M. J. Kortelainen, ${ }^{161}$ S. Lammel, ${ }^{161}$ J. Lewis, ${ }^{161}$ D. Lincoln, ${ }^{161}$ R. Lipton, ${ }^{161}$ M. Liu, ${ }^{161}$ T. Liu, ${ }^{161}$ J. Lykken, ${ }^{161}$ K. Maeshima, ${ }^{161}$ D. Mason, ${ }^{161}$ P. McBride, ${ }^{161}$ P. Merkel, ${ }^{161}$ S. Mrenna, ${ }^{161}$ S. Nahn, ${ }^{161}$ V. O’Dell ${ }^{161}$ V. Papadimitriou, ${ }^{161}$ K. Pedro, ${ }^{161}$ C. Pena, ${ }^{161, y y}$ O. Prokofyev, ${ }^{161}$ F. Ravera, ${ }^{161}$ A. Reinsvold Hall, ${ }^{161}$ L. Ristori, ${ }^{161}$ B. Schneider, ${ }^{161}$ E. Sexton-Kennedy, ${ }^{161}$ N. Smith, ${ }^{161}$ A. Soha ${ }^{161}$ W. J. Spalding, ${ }^{161}$ L. Spiegel, ${ }^{161}$ S. Stoynev, ${ }^{161}$ J. Strait, ${ }^{161}$ L. Taylor, ${ }^{161}$ S. Tkaczyk, ${ }^{161}$ N. V. Tran, ${ }^{161}$ L. Uplegger, ${ }^{161}$ E. W. Vaandering, ${ }^{161}$ M. Wang, ${ }^{161}$ H. A. Weber,${ }^{161}$ A. Woodard, ${ }^{161}$ D. Acosta, ${ }^{162}$ P. Avery, ${ }^{162}$ D. Bourilkov, ${ }^{162}$ L. Cadamuro, ${ }^{162}$ V. Cherepanov, ${ }^{162}$ F. Errico, ${ }^{162}$ R. D. Field, ${ }^{162}$ D. Guerrero, ${ }^{162}$ B. M. Joshi, ${ }^{162}$ M. Kim, ${ }^{162}$ J. Konigsberg, ${ }^{162}$ A. Korytov, ${ }^{162}$ K. H. Lo, ${ }^{162}$ K. Matchev, ${ }^{162}$ N. Menendez, ${ }^{162}$

G. Mitselmakher, ${ }^{162}$ D. Rosenzweig, ${ }^{162}$ K. Shi, ${ }^{162}$ J. Wang, ${ }^{162}$ S. Wang, ${ }^{162}$ X. Zuo, ${ }^{162}$ Y. R. Joshi, ${ }^{163}$ T. Adams, ${ }^{164}$ A. Askew, ${ }^{164}$ D. Diaz, ${ }^{164}$ R. Habibullah, ${ }^{164}$ S. Hagopian, ${ }^{164}$ V. Hagopian, ${ }^{164}$ K. F. Johnson, ${ }^{164}$ R. Khurana, ${ }^{164}$ T. Kolberg, ${ }^{164}$ G. Martinez, ${ }^{164}$ H. Prosper, ${ }^{164}$ C. Schiber, ${ }^{164}$ R. Yohay, ${ }^{164}$ J. Zhang, ${ }^{164}$ M. M. Baarmand ${ }^{165}$ S. Butalla, ${ }^{165}$ T. Elkafrawy, ${ }^{165, n}$ M. Hohlmann, ${ }^{165}$ D. Noonan, ${ }^{165}$ M. Rahmani, ${ }^{165}$ M. Saunders, ${ }^{165}$ F. Yumiceva, ${ }^{165}$ M. R. Adams, ${ }^{166}$ L. Apanasevich, ${ }^{166}$ H. Becerril Gonzalez, ${ }^{166}$ R. Cavanaugh, ${ }^{166}$ X. Chen, ${ }^{166}$ S. Dittmer, ${ }^{166}$ O. Evdokimov, ${ }^{166}$ C. E. Gerber ${ }^{166}$ D. A. Hangal, ${ }^{166}$ D. J. Hofman, ${ }^{166}$ C. Mills, ${ }^{166}$ G. Oh, ${ }^{166}$ T. Roy,${ }^{166}$ M. B. Tonjes, ${ }^{166}$ N. Varelas, ${ }^{166}$ J. Viinikainen, ${ }^{166}$ H. Wang, ${ }^{166}$ X. Wang, ${ }^{166}$ Z. Wu, ${ }^{166}$ M. Alhusseini, ${ }^{167}$ B. Bilki, ${ }^{167,111}$ K. Dilsiz, ${ }^{167, \text { ffff }}$ S. Durgut, ${ }^{167}$ R. P. Gandrajula, ${ }^{167}$ M. Haytmyradov, ${ }^{167}$ V. Khristenko, ${ }^{167}$ O. K. Köseyan, ${ }^{167}$ J.-P. Merlo, ${ }^{167}$ A. Mestvirishvili, ${ }^{167, g g g g}$ A. Moeller, ${ }^{167}$ J. Nachtman, ${ }^{167}$ H. Ogul, ${ }^{167, \text { hhhh }}$ Y. Onel, ${ }^{167}$ F. Ozok, ${ }^{167, \text { iiii }}$ A. Penzo, ${ }^{167}$ C. Snyder,${ }^{167}$ E. Tiras, ${ }^{167}$ J. Wetzel, ${ }^{167}$ K. Yi, ${ }^{167, j j j j}$ O. Amram, ${ }^{168}$ B. Blumenfeld,${ }^{168}$ L. Corcodilos, ${ }^{168}$ M. Eminizer, ${ }^{168}$ A. V. Gritsan, ${ }^{168}$ S. Kyriacou, ${ }^{168}$ P. Maksimovic, ${ }^{168}$ C. Mantilla, ${ }^{168}$ J. Roskes, ${ }^{168}$ M. Swartz ${ }^{168}$ T. Á. Vámi, ${ }^{168}$ C. Baldenegro Barrera, ${ }^{169}$ P. Baringer, ${ }^{169}$ A. Bean, ${ }^{169}$ A. Bylinkin, ${ }^{169}$ T. Isidori, ${ }^{169}$ S. Khalil, ${ }^{169}$ J. King, ${ }^{169}$ G. Krintiras, ${ }^{169}$ A. Kropivnitskaya, ${ }^{169}$ C. Lindsey, ${ }^{169}$ N. Minafra, ${ }^{169}$ M. Murray, ${ }^{169}$ C. Rogan, ${ }^{169}$ C. Royon, ${ }^{169}$ S. Sanders, ${ }^{169}$ E. Schmitz, ${ }^{169}$ J. D. Tapia Takaki, ${ }^{169}$ Q. Wang, ${ }^{169}$ J. Williams, ${ }^{169}$ G. Wilson, ${ }^{169}$ S. Duric, ${ }^{170}$ A. Ivanov, ${ }^{170}$ K. Kaadze, ${ }^{170}$ D. Kim, ${ }^{170}$ Y. Maravin, ${ }^{170}$ D. R. Mendis, ${ }^{170}$ T. Mitchell, ${ }^{170}$ A. Modak, ${ }^{170}$ A. Mohammadi, ${ }^{170}$ F. Rebassoo, ${ }^{171}$ D. Wright, ${ }^{171}$ E. Adams, ${ }^{172}$ A. Baden, ${ }^{172}$ O. Baron, ${ }^{172}$ A. Belloni, ${ }^{172}$ S. C. Eno, ${ }^{172}$ Y. Feng, ${ }^{172}$ N. J. Hadley, ${ }^{172}$ S. Jabeen, ${ }^{172}$ G. Y. Jeng, ${ }^{172}$ R. G. Kellogg, ${ }^{172}$ T. Koeth, ${ }^{172}$ A. C. Mignerey, ${ }^{172}$ S. Nabili, ${ }^{172}$ M. Seidel,${ }^{172}$ A. Skuja, ${ }^{172}$ S. C. Tonwar, ${ }^{172}$ L. Wang, ${ }^{172}$ K. Wong, ${ }^{172}$ D. Abercrombie, ${ }^{173}$ B. Allen, ${ }^{173}$ R. Bi, ${ }^{173}$ S. Brandt, ${ }^{173}$ W. Busza, ${ }^{173}$ I. A. Cali, ${ }^{173}$ Y. Chen, ${ }^{173}$ M. D’Alfonso, ${ }^{173}$ G. Gomez Ceballos, ${ }^{173}$ M. Goncharov, ${ }^{173}$ P. Harris,${ }^{173}$ D. Hsu,${ }^{173}$ M. Hu, ${ }^{173}$ M. Klute, ${ }^{173}$ D. Kovalskyi, ${ }^{173}$ J. Krupa,${ }^{173}$ Y.-J. Lee, ${ }^{173}$ P. D. Luckey, ${ }^{173}$ B. Maier, ${ }^{173}$ A. C. Marini, ${ }^{173}$ C. Mcginn, ${ }^{173}$ C. Mironov, ${ }^{173}$ S. Narayanan, ${ }^{173}$ X. Niu, ${ }^{173}$ C. Paus, ${ }^{173}$ D. Rankin, ${ }^{173}$ C. Roland, ${ }^{173}$ G. Roland, ${ }^{173}$ Z. Shi, ${ }^{173}$ G. S. F. Stephans, ${ }^{173}$ K. Sumorok, ${ }^{173}$ K. Tatar, ${ }^{173}$ D. Velicanu, ${ }^{173}$ J. Wang, ${ }^{173}$ T. W. Wang, ${ }^{173}$ Z. Wang, ${ }^{173}$ B. Wyslouch, ${ }^{173}$ R. M. Chatterjee, ${ }^{174}$ A. Evans, ${ }^{174}$ S. Guts, ${ }^{174, a}$ P. Hansen, ${ }^{174}$ J. Hiltbrand, ${ }^{174}$ Sh. Jain, ${ }^{174}$ M. Krohn, ${ }^{174}$ Y. Kubota, ${ }^{174}$ Z. Lesko, ${ }^{174}$ J. Mans, ${ }^{174}$ M. Revering, ${ }^{174}$ R. Rusack, ${ }^{174}$ 
R. Saradhy, ${ }^{174}$ N. Schroeder, ${ }^{174}$ N. Strobbe,${ }^{174}$ M. A. Wadud, ${ }^{174}$ J. G. Acosta, ${ }^{175}$ S. Oliveros,${ }^{175}$ K. Bloom, ${ }^{176}$ S. Chauhan, ${ }^{176}$ D. R. Claes, ${ }^{176}$ C. Fangmeier, ${ }^{176}$ L. Finco,${ }^{176}$ F. Golf, ${ }^{176}$ J. R. González Fernández, ${ }^{176}$ I. Kravchenko, ${ }^{176}$ J. E. Siado, ${ }^{176}$

G. R. Snow, ${ }^{176, a}$ B. Stieger, ${ }^{176}$ W. Tabb, ${ }^{176}$ G. Agarwal, ${ }^{177}$ C. Harrington, ${ }^{177}$ L. Hay, ${ }^{177}$ I. Iashvili, ${ }^{177}$ A. Kharchilava, ${ }^{177}$ C. McLean, ${ }^{177}$ D. Nguyen, ${ }^{177}$ A. Parker, ${ }^{177}$ J. Pekkanen, ${ }^{177}$ S. Rappoccio, ${ }^{177}$ B. Roozbahani, ${ }^{177}$ G. Alverson, ${ }^{178}$ E. Barberis ${ }^{178}$ C. Freer, ${ }^{178}$ Y. Haddad, ${ }^{178}$ A. Hortiangtham,${ }^{178}$ G. Madigan, ${ }^{178}$ B. Marzocchi, ${ }^{178}$ D. M. Morse,${ }^{178}$ V. Nguyen, ${ }^{178}$ T. Orimoto, ${ }^{178}$ L. Skinnari, ${ }^{178}$ A. Tishelman-Charny, ${ }^{178}$ T. Wamorkar, ${ }^{178}$ B. Wang, ${ }^{178}$ A. Wisecarver, ${ }^{178}$ D. Wood, ${ }^{178}$ S. Bhattacharya, ${ }^{179}$ J. Bueghly, ${ }^{179}$ Z. Chen, ${ }^{179}$ A. Gilbert, ${ }^{179}$ T. Gunter, ${ }^{179}$ K. A. Hahn, ${ }^{179}$ N. Odell, ${ }^{179}$ M. H. Schmitt, ${ }^{179}$ K. Sung, ${ }^{179}$ M. Velasco, ${ }^{179}$ R. Bucci, ${ }^{180}$ N. Dev,${ }^{180}$ R. Goldouzian, ${ }^{180}$ M. Hildreth, ${ }^{180}$

K. Hurtado Anampa ${ }^{180}$ C. Jessop, ${ }^{180}$ D. J. Karmgard, ${ }^{180}$ K. Lannon, ${ }^{180}$ W. Li, ${ }^{180}$ N. Loukas, ${ }^{180}$ N. Marinelli, ${ }^{180}$ I. Mcalister, ${ }^{180}$ F. Meng, ${ }^{180}$ K. Mohrman, ${ }^{180}$ Y. Musienko, ${ }^{180, s s}$ R. Ruchti, ${ }^{180}$ P. Siddireddy, ${ }^{180}$ S. Taroni, ${ }^{180}$ M. Wayne, ${ }^{180}$ A. Wightman, ${ }^{180}$ M. Wolf, ${ }^{180}$ L. Zygala, ${ }^{180}$ J. Alimena, ${ }^{181}$ B. Bylsma, ${ }^{181}$ B. Cardwell ${ }^{181}$ L. S. Durkin, ${ }^{181}$ B. Francis, ${ }^{181}$ C. Hill, ${ }^{181}$ W. Ji, ${ }^{181}$ A. Lefeld, ${ }^{181}$ B. L. Winer, ${ }^{181}$ B. R. Yates, ${ }^{181}$ G. Dezoort, ${ }^{182}$ P. Elmer, ${ }^{182}$ B. Greenberg, ${ }^{182}$ N. Haubrich, ${ }^{182}$ S. Higginbotham, ${ }^{182}$ A. Kalogeropoulos, ${ }^{182}$ G. Kopp, ${ }^{182}$ S. Kwan, ${ }^{182}$ D. Lange, ${ }^{182}$ M. T. Lucchini, ${ }^{182}$ J. Luo, ${ }^{182}$ D. Marlow, ${ }^{182}$ K. Mei, ${ }^{182}$ I. Ojalvo, ${ }^{182}$ J. Olsen, ${ }^{182}$ C. Palmer, ${ }^{182}$ P. Piroué, ${ }^{182}$ D. Stickland, ${ }^{182}$ C. Tully, ${ }^{182}$ S. Malik, ${ }^{183}$ S. Norberg, ${ }^{183}$ V. E. Barnes, ${ }^{184}$ R. Chawla, ${ }^{184}$ S. Das, ${ }^{184}$ L. Gutay, ${ }^{184}$ M. Jones, ${ }^{184}$ A. W. Jung, ${ }^{184}$ B. Mahakud, ${ }^{184}$ G. Negro, ${ }^{184}$ N. Neumeister, ${ }^{184}$ C. C. Peng,${ }^{184}$ S. Piperov,${ }^{184}$ H. Qiu, ${ }^{184}$ J. F. Schulte, ${ }^{184}$ N. Trevisani, ${ }^{184}$ F. Wang, ${ }^{184}$ R. Xiao, ${ }^{184}$ W. Xie, ${ }^{184}$ T. Cheng, ${ }^{185}$ J. Dolen, ${ }^{185}$ N. Parashar, ${ }^{185}$ M. Stojanovic, ${ }^{185}$ A. Baty, ${ }^{186}$ S. Dildick, ${ }^{186}$ K. M. Ecklund, ${ }^{186}$ S. Freed, ${ }^{186}$ F. J. M. Geurts, ${ }^{186}$ M. Kilpatrick, ${ }^{186}$ A. Kumar, ${ }^{186}$ W. Li ${ }^{186}$ B. P. Padley, ${ }^{186}$ R. Redjimi ${ }^{186}$ J. Roberts, ${ }^{186, a}$ J. Rorie, ${ }^{186}$ W. Shi, ${ }^{186}$ A. G. Stahl Leiton, ${ }^{186}$ Z. Tu, ${ }^{186}$ A. Zhang, ${ }^{186}$ A. Bodek, ${ }^{187}$ P. de Barbaro, ${ }^{187}$ R. Demina, ${ }^{187}$ J. L. Dulemba, ${ }^{187}$ C. Fallon, ${ }^{187}$ T. Ferbel,${ }^{187}$ M. Galanti, ${ }^{187}$ A. Garcia-Bellido, ${ }^{187}$ O. Hindrichs, ${ }^{187}$ A. Khukhunaishvili, ${ }^{187}$ E. Ranken, ${ }^{187}$ R. Taus, ${ }^{187}$ B. Chiarito, ${ }^{188}$ J. P. Chou, ${ }^{188}$ A. Gandrakota, ${ }^{188}$ Y. Gershtein, ${ }^{188}$ E. Halkiadakis,${ }^{188}$ A. Hart, ${ }^{188}$ M. Heindl, ${ }^{188}$ E. Hughes ${ }^{188}$ S. Kaplan, ${ }^{188}$ O. Karacheban, ${ }^{188, x}$ I. Laflotte, ${ }^{188}$ A. Lath ${ }^{188}$ R. Montalvo, ${ }^{188}$ K. Nash, ${ }^{188}$ M. Osherson, ${ }^{188}$ S. Salur, ${ }^{188}$ S. Schnetzer, ${ }^{188}$ S. Somalwar, ${ }^{188}$ R. Stone, ${ }^{188}$ S. A. Thayil,${ }^{188}$ S. Thomas ${ }^{188}$ H. Acharya,${ }^{189}$ A. G. Delannoy, ${ }^{189}$ S. Spanier, ${ }^{189}$ O. Bouhali, ${ }^{190, k k k k}$ M. Dalchenko, ${ }^{190}$ A. Delgado,${ }^{190}$ R. Eusebi, ${ }^{190}$ J. Gilmore,${ }^{190}$ T. Huang, ${ }^{190}$ T. Kamon, ${ }^{190,1111}$ H. Kim, ${ }^{190}$ S. Luo, ${ }^{190}$ S. Malhotra, ${ }^{190}$ R. Mueller, ${ }^{190}$ D. Overton, ${ }^{190}$ L. Perniè, ${ }^{190}$ D. Rathjens, ${ }^{190}$ A. Safonov, ${ }^{190}$ N. Akchurin, ${ }^{191}$ J. Damgov, ${ }^{191}$ V. Hegde,${ }^{191}$ S. Kunori, ${ }^{191}$ K. Lamichhane,${ }^{191}$ S. W. Lee,${ }^{191}$ T. Mengke, ${ }^{191}$ S. Muthumuni, ${ }^{191}$ T. Peltola, ${ }^{191}$ S. Undleeb, ${ }^{191}$ I. Volobouev, ${ }^{191}$ Z. Wang, ${ }^{191}$ A. Whitbeck, ${ }^{191}$ E. Appelt, ${ }^{192}$ S. Greene, ${ }^{192}$ A. Gurrola, ${ }^{192}$ R. Janjam, ${ }^{192}$ W. Johns, ${ }^{192}$ C. Maguire, ${ }^{192}$ A. Melo, ${ }^{192}$ H. Ni, ${ }^{192}$ K. Padeken, ${ }^{192}$ F. Romeo, ${ }^{192}$ P. Sheldon, ${ }^{192}$ S. Tuo,${ }^{192}$ J. Velkovska ${ }^{192}$ M. Verweij ${ }^{192}$ L. Ang, ${ }^{193}$ M. W. Arenton, ${ }^{193}$ B. Cox ${ }^{193}$ G. Cummings,${ }^{193}$ J. Hakala, ${ }^{193}$ R. Hirosky, ${ }^{193}$ M. Joyce, ${ }^{193}$ A. Ledovskoy, ${ }_{193}$ C. Neu, ${ }^{193}$ B. Tannenwald, ${ }^{193}$ Y. Wang, ${ }^{193}$ E. Wolfe, ${ }^{193}$ F. Xia, ${ }^{193}$ P. E. Karchin,${ }^{194}$ N. Poudyal, ${ }^{194}$ J. Sturdy,${ }^{194}$ P. Thapa, ${ }^{194}$ K. Black,,${ }^{195}$ T. Bose,${ }^{195}$ J. Buchanan,,${ }^{195}$ C. Caillol, ${ }^{195}$ S. Dasu, ${ }^{195}$ I. De Bruyn, ${ }^{195}$ L. Dodd, ${ }^{195}$ C. Galloni, ${ }^{195}$ H. He,${ }^{195}$ M. Herndon, ${ }^{195}$ A. Hervé,${ }^{195}$ U. Hussain, ${ }^{195}$ A. Lanaro, ${ }^{195}$ A. Loeliger, ${ }^{195}$ R. Loveless, ${ }^{195}$ J. Madhusudanan Sreekala, ${ }^{195}$ A. Mallampalli, ${ }^{195}$ D. Pinna, ${ }^{195}$ T. Ruggles, ${ }^{195}$ A. Savin, ${ }^{195}$ V. Shang, ${ }^{195}$ V. Sharma, ${ }^{195}$ W. H. Smith, ${ }^{195}$ D. Teague, ${ }^{195}$ S. Trembath-reichert, ${ }^{195}$ and W. Vetens ${ }^{195}$

(CMS Collaboration)

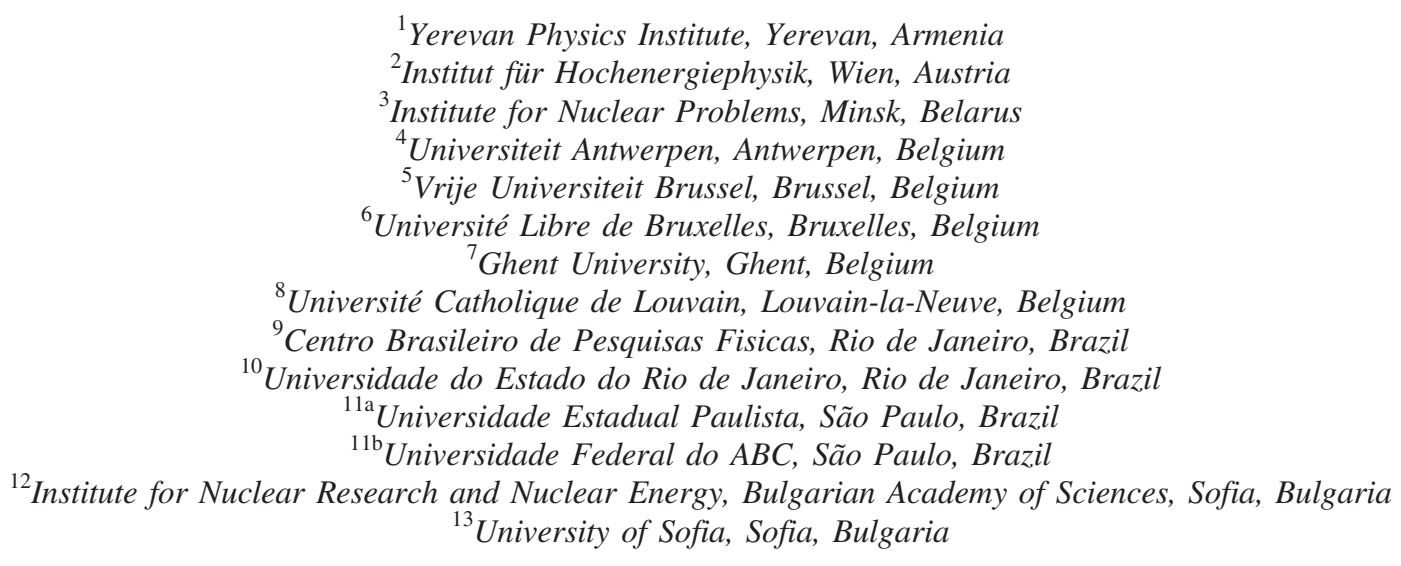




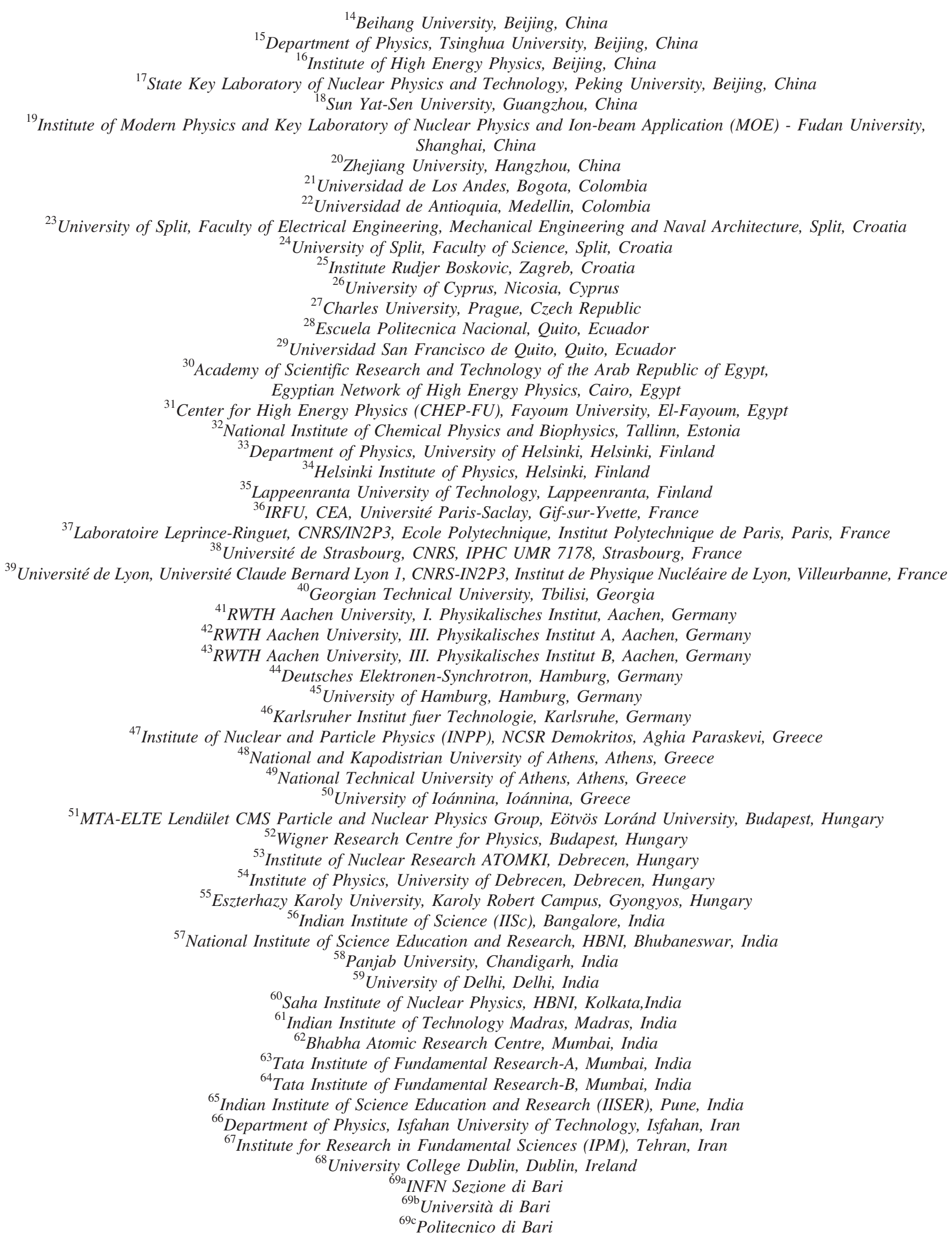


${ }^{70 a}$ INFN Sezione di Bologna, Bologna, Italy

${ }^{70 \mathrm{~b}}$ Università di Bologna, Bologna, Italy

${ }^{71 a}$ INFN Sezione di Catania, Catania, Italy

${ }^{71 \mathrm{~b}}$ Università di Catania, Catania, Italy

${ }^{72 \mathrm{a}}$ INFN Sezione di Firenze, Firenze, Italy

${ }^{72 \mathrm{~b}}$ Università di Firenze, Firenze, Italy

${ }^{73}$ INFN Laboratori Nazionali di Frascati, Frascati, Italy

${ }^{74 a}$ INFN Sezione di Genova, Genova, Italy

${ }^{74 \mathrm{~b}}$ Università di Genova, Genova, Italy

${ }^{75 a}$ INFN Sezione di Milano-Bicocca, Milano, Italy

${ }^{75 b}$ Università di Milano-Bicocca, Milano, Italy

${ }^{76 a}$ INFN Sezione di Napoli, Napoli, Italy

${ }^{76 \mathrm{~b}}$ Università di Napoli 'Federico II', Napoli, Italy

${ }^{76 c}$ Università della Basilicata, Potenza, Italy

${ }^{76 \mathrm{~d}}$ Università G. Marconi, Roma, Italy

${ }^{77}$ INFN Sezione di Padova, Padova, Italy

${ }^{77 b}$ Università di Padova, Padova, Italy

${ }^{77 \mathrm{c}}$ Università di Trento, Trento, Italy

${ }^{78}$ INFN Sezione di Pavia

${ }^{78 b}$ Università di Pavia

${ }^{79 a}$ INFN Sezione di Perugia, Perugia, Italy

${ }^{79 b}$ Università di Perugia, Perugia, Italy

${ }^{80 a}$ INFN Sezione di Pisa, Pisa, Italy

${ }^{80 \mathrm{~b}}$ Università di Pisa, Pisa, Italy

${ }^{80 \mathrm{c}}$ Scuola Normale Superiore di Pisa, Pisa, Italy

${ }^{81 \mathrm{a}}$ INFN Sezione di Roma, Rome, Italy

${ }^{81 \mathrm{~b}}$ Sapienza Università di Roma, Rome, Italy

${ }^{82 \mathrm{a}}$ INFN Sezione di Torinos, Torino, Italy

${ }^{82 \mathrm{~b}}$ Università di Torino, Torino, Italy

${ }^{82 \mathrm{c}}$ Università del Piemonte Orientale, Novara, Italy

${ }^{83 a}$ INFN Sezione di Trieste, Trieste, Italy

${ }^{83 \mathrm{~b}}$ Università di Trieste, Trieste, Italy

${ }^{84}$ Kyungpook National University, Daegu, Korea

${ }^{85}$ Chonnam National University, Institute for Universe and Elementary Particles, Kwangju, Korea

${ }^{86}$ Hanyang University, Seoul, Korea

${ }^{87}$ Korea University, Seoul, Korea

${ }^{88}$ Kyung Hee University, Department of Physics, Seoul, Republic of Korea

${ }^{89}$ Sejong University, Seoul, Korea

${ }^{90}$ Seoul National University, Seoul, Korea

${ }^{91}$ University of Seoul, Seoul, Korea

${ }^{92}$ Yonsei University, Department of Physics, Seoul, Korea

${ }^{93}$ Sungkyunkwan University, Suwon, Korea

${ }^{94}$ Riga Technical University, Riga, Latvia

${ }^{95}$ Vilnius University, Vilnius, Lithuania

${ }^{96}$ National Centre for Particle Physics, Universiti Malaya, Kuala Lumpur, Malaysia

${ }^{97}$ Universidad de Sonora (UNISON), Hermosillo, Mexico

${ }^{98}$ Centro de Investigacion y de Estudios Avanzados del IPN, Mexico City, Mexico

${ }^{99}$ Universidad Iberoamericana, Mexico City, Mexico

${ }^{100}$ Benemerita Universidad Autonoma de Puebla, Puebla, Mexico

${ }^{101}$ Universidad Autónoma de San Luis Potosí, San Luis Potosí, Mexico

${ }^{102}$ University of Montenegro, Podgorica, Montenegro

${ }^{103}$ University of Auckland, Auckland, New Zealand

${ }^{104}$ University of Canterbury, Christchurch, New Zealand

${ }^{105}$ National Centre for Physics, Quaid-I-Azam University, Islamabad, Pakistan

${ }^{106}$ AGH University of Science and Technology Faculty of Computer Science, Electronics and Telecommunications, Krakow, Poland

${ }^{107}$ National Centre for Nuclear Research, Swierk, Poland

${ }^{108}$ Institute of Experimental Physics, Faculty of Physics, University of Warsaw, Warsaw, Poland

${ }^{109}$ Laboratório de Instrumentação e Física Experimental de Partículas, Lisboa, Portugal

${ }^{110}$ Joint Institute for Nuclear Research, Dubna, Russia

${ }^{111}$ Petersburg Nuclear Physics Institute, Gatchina (St. Petersburg), Russia 
${ }^{112}$ Institute for Nuclear Research, Moscow, Russia

${ }^{113}$ Institute for Theoretical and Experimental Physics named by A.I. Alikhanov of NRC 'Kurchatov Institute', Moscow, Russia

${ }^{114}$ Moscow Institute of Physics and Technology, Moscow, Russia

${ }^{115}$ National Research Nuclear University 'Moscow Engineering Physics Institute' (MEPhI), Moscow, Russia

${ }^{116}$ P.N. Lebedev Physical Institute, Moscow, Russia

${ }^{117}$ Skobeltsyn Institute of Nuclear Physics, Lomonosov Moscow State University, Moscow, Russia

${ }^{118}$ Novosibirsk State University (NSU), Novosibirsk, Russia

${ }^{119}$ Institute for High Energy Physics of National Research Centre 'Kurchatov Institute', Protvino, Russia

${ }^{120}$ National Research Tomsk Polytechnic University, Tomsk, Russia

${ }^{121}$ Tomsk State University, Tomsk, Russia

${ }^{122}$ University of Belgrade: Faculty of Physics and VINCA Institute of Nuclear Sciences, Belgrade, Serbia

${ }^{123}$ Centro de Investigaciones Energéticas Medioambientales y Tecnológicas (CIEMAT), Madrid, Spain

${ }^{124}$ Universidad Autónoma de Madrid, Madrid, Spain

${ }^{125}$ Universidad de Oviedo, Instituto Universitario de Ciencias y Tecnologías Espaciales de Asturias (ICTEA), Oviedo, Spain

${ }^{126}$ Instituto de Física de Cantabria (IFCA), CSIC-Universidad de Cantabria, Santander, Spain

${ }^{127}$ University of Colombo, Colombo, Sri Lanka

${ }^{128}$ University of Ruhuna, Department of Physics, Matara, Sri Lanka

${ }^{129}$ CERN, European Organization for Nuclear Research, Geneva, Switzerland

${ }^{130}$ Paul Scherrer Institut, Villigen, Switzerland

${ }^{131}$ ETH Zurich-Institute for Particle Physics and Astrophysics (IPA), Zurich, Switzerland

${ }^{132}$ Universität Zürich, Zurich, Switzerland

${ }^{133}$ National Central University, Chung-Li, Taiwan

${ }^{134}$ National Taiwan University (NTU), Taipei, Taiwan

${ }^{135}$ Chulalongkorn University, Faculty of Science, Department of Physics, Bangkok, Thailand

${ }^{136}$ Cukurova University, Physics Department, Science and Art Faculty, Adana, Turkey

${ }^{137}$ Middle East Technical University, Physics Department, Ankara, Turkey

${ }^{138}$ Bogazici University, Istanbul, Turkey

${ }^{139}$ Istanbul Technical University, Istanbul, Turkey

${ }^{140}$ Istanbul University, Istanbul, Turkey

${ }^{141}$ Institute for Scintillation Materials of National Academy of Science of Ukraine, Kharkov, Ukraine

${ }^{142}$ National Scientific Center, Kharkov Institute of Physics and Technology, Kharkov, Ukraine

${ }^{143}$ University of Bristol, Bristol, United Kingdom

${ }^{144}$ Rutherford Appleton Laboratory, Didcot, United Kingdom

${ }^{145}$ Imperial College, London, United Kingdom

${ }^{146}$ Brunel University, Uxbridge, United Kingdom

${ }^{147}$ Baylor University, Waco, Texas, USA

${ }^{148}$ Catholic University of America, Washington, DC, USA

${ }^{149}$ The University of Alabama, Tuscaloosa, Alabama, USA

${ }^{150}$ Boston University, Boston, Massachusetts, USA

${ }^{151}$ Brown University, Providence, Rhode Island, USA

${ }^{152}$ University of California, Davis, Davis, California, USA

${ }^{153}$ University of California, Los Angeles, California, USA

${ }^{154}$ University of California, Riverside, Riverside, California, USA

${ }^{155}$ University of California, San Diego, La Jolla, California, USA

${ }^{156}$ University of California, Santa Barbara - Department of Physics, Santa Barbara, California, USA

${ }^{157}$ California Institute of Technology, Pasadena, California, USA

${ }^{158}$ Carnegie Mellon University, Pittsburgh, Pennsylvania, USA

${ }^{159}$ University of Colorado Boulder, Boulder, Colorado, USA

${ }^{160}$ Cornell University, Ithaca, New York, USA

${ }^{161}$ Fermi National Accelerator Laboratory, Batavia, Illinois, USA

${ }^{162}$ University of Florida, Gainesville, Florida, USA

${ }^{163}$ Florida International University, Miami, Florida, USA

${ }^{164}$ Florida State University, Tallahassee, Florida, USA

${ }^{165}$ Florida Institute of Technology, Melbourne, Florida, USA

${ }^{166}$ University of Illinois at Chicago (UIC), Chicago, Illinois, USA

${ }^{167}$ The University of Iowa, Iowa City, Iowa, USA

${ }^{168}$ Johns Hopkins University, Baltimore, Maryland, USA

${ }^{169}$ The University of Kansas, Lawrence, Kansas, USA

${ }^{170}$ Kansas State University, Manhattan, Kansas, USA

${ }^{171}$ Lawrence Livermore National Laboratory, Livermore, California, USA 


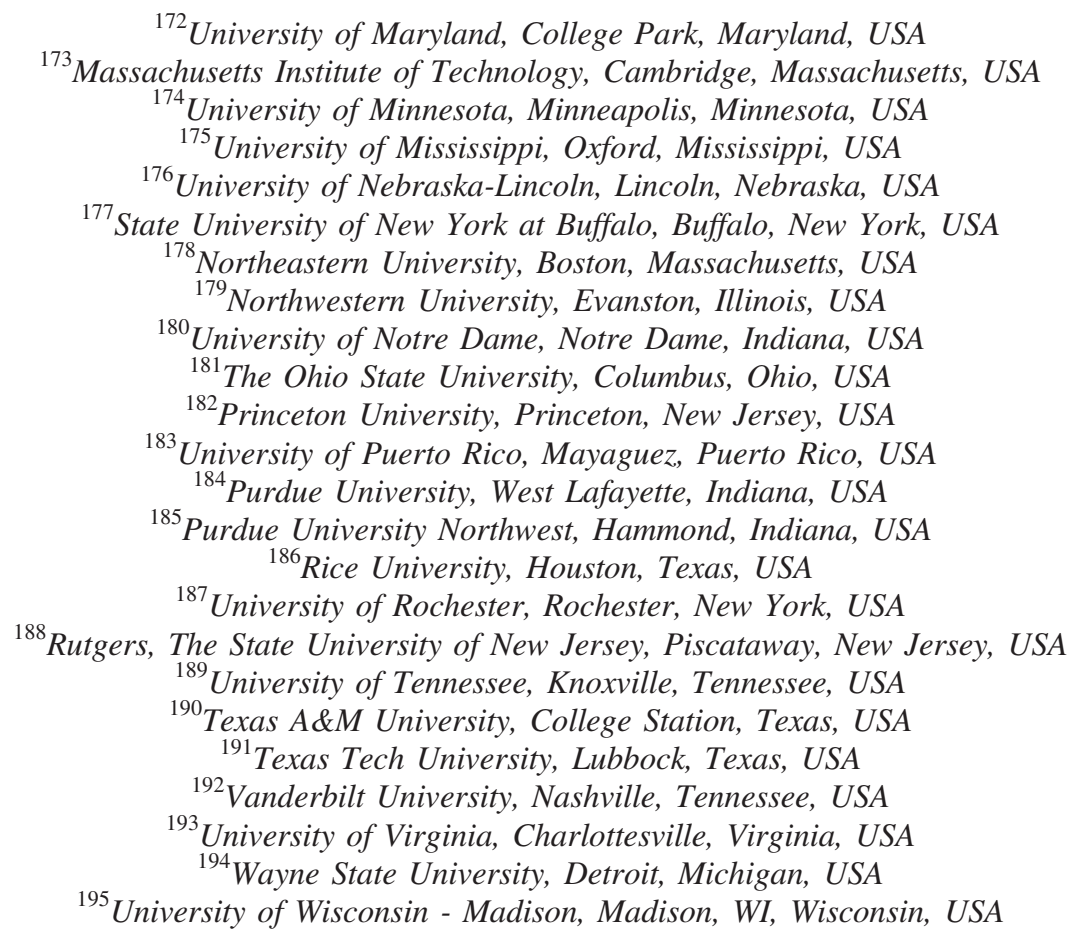

${ }^{\mathrm{a}}$ Deceased.

${ }^{\mathrm{b}}$ Also at Vienna University of Technology, Vienna, Austria

${ }^{c}$ Also at Department of Basic and Applied Sciences, Faculty of Engineering, Arab Academy for Science, Technology and Maritime Transport

${ }^{\mathrm{d}}$ Also at Université Libre de Bruxelles, Bruxelles, Belgium

${ }^{\mathrm{e}}$ Also at IRFU, CEA, Université Paris-Saclay, Gif-sur-Yvette, France

${ }_{\mathrm{f}}^{\mathrm{f}}$ Also at Universidade Estadual de Campinas, Campinas, Brazil

${ }^{\mathrm{g}}$ Also at Federal University of Rio Grande do Sul, Porto Alegre, Brazil

${ }^{\mathrm{h}}$ Also at UFMS

${ }^{\mathrm{i}}$ Also at Universidade Federal de Pelotas, Pelotas, Brazil

${ }^{\mathrm{j}}$ Also at University of Chinese Academy of Sciences

${ }^{\mathrm{k}}$ Also at Institute for Theoretical and Experimental Physics named by A.I. Alikhanov of NRC 'Kurchatov Institute', Moscow, Russia

${ }^{1}$ Also at Joint Institute for Nuclear Research, Dubna, Russia

${ }^{\mathrm{m}}$ Also at Zewail City of Science and Technology, Zewail, Egypt

${ }^{\mathrm{n}}$ Also at Ain Shams University, Cairo, Egypt

${ }^{\circ}$ Also at British University in Egypt, Cairo, Egypt

${ }^{\mathrm{p}}$ Also at Purdue University, West Lafayette, Indiana, USA

${ }^{\mathrm{q}}$ Also at Université de Haute Alsace, Mulhouse, France

${ }^{\mathrm{r}}$ Also at Tbilisi State University, Tbilisi, Georgia

${ }^{\mathrm{s}}$ Also at Erzincan Binali Yildirim University, Erzincan, Turkey

${ }^{\mathrm{t}}$ Also at CERN, European Organization for Nuclear Research, Geneva, Switzerland

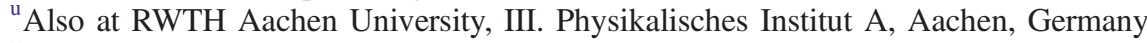

${ }^{v}$ Also at University of Hamburg, Hamburg, Germany

${ }^{\mathrm{w}}$ Also at Department of Physics, Isfahan University of Technology, Isfahan, Iran

${ }^{\mathrm{x}}$ Also at Brandenburg University of Technology, Cottbus, Germany

${ }^{y}$ Also at Skobeltsyn Institute of Nuclear Physics, Lomonosov Moscow State University, Moscow, Russia

${ }^{\mathrm{z}}$ Also at Institute of Physics, University of Debrecen, Debrecen, Hungary

${ }^{\text {aa }}$ Also at Physics Department, Faculty of Science, Assiut University

${ }^{\mathrm{bb}}$ Also at MTA-ELTE Lendület CMS Particle and Nuclear Physics Group, Eötvös Loránd University, Budapest, Hungary

${ }^{c c}$ Also at Institute of Nuclear Research ATOMKI, Debrecen, Hungary

${ }^{\mathrm{dd}}$ Also at IIT Bhubaneswar, Bhubaneswar, India

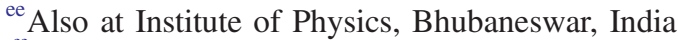

${ }^{\mathrm{ff}}$ Also at G.H.G. Khalsa College, Punjab, India

${ }^{g g}$ Also at Shoolini University, Solan, India

${ }^{\text {hh }}$ Also at University of Hyderabad, Hyderabad, India 


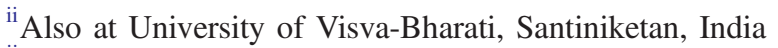

${ }^{\mathrm{jj}}$ Also at Indian Institute of Technology (IIT), Mumbai, India

${ }^{\mathrm{kk}}$ Also at Deutsches Elektronen-Synchrotron, Hamburg, Germany

${ }^{11}$ Also at Department of Physics, University of Science and Technology of Mazandaran, Behshahr, Iran

${ }^{\mathrm{mm}}$ Also at INFN Sezione di Bari, Università di Bari, Politecnico di Bari, Bari, Italy

${ }^{n n}$ Also at Italian National Agency for New Technologies, Energy and Sustainable Economic Development

${ }^{0 o}$ Also at Centro Siciliano di Fisica Nucleare e di Struttura Della Materia

${ }^{\mathrm{pp}}$ Also at Riga Technical University, Riga, Latvia

${ }^{\mathrm{qq}}$ Also at Consejo Nacional de Ciencia y Tecnología, Mexico City, Mexico

${ }^{\text {rr }}$ Also at Warsaw University of Technology, Institute of Electronic Systems, Warsaw, Poland

${ }^{\text {ss }}$ Also at Institute for Nuclear Research, Moscow, Russia

${ }^{\text {tt} A l s o ~ a t ~ N a t i o n a l ~ R e s e a r c h ~ N u c l e a r ~ U n i v e r s i t y ~ ' M o s c o w ~ E n g i n e e r i n g ~ P h y s i c s ~ I n s t i t u t e ' ~(M E P h I), ~ M o s c o w, ~ R u s s i a ~}$

${ }^{u u}$ Also at St. Petersburg State Polytechnical University, St. Petersburg, Russia

${ }^{\mathrm{vv}}$ Also at University of Florida, Gainesville, Florida, USA

${ }^{\text {ww }}$ Also at Imperial College, London, United Kingdom

${ }^{\mathrm{xx}}$ Also at P.N. Lebedev Physical Institute, Moscow, Russia

${ }^{y y}$ Also at California Institute of Technology, Pasadena, California, USA

${ }^{\mathrm{zz}}$ Also at Budker Institute of Nuclear Physics, Novosibirsk, Russia

${ }^{\text {aaa }}$ Also at Faculty of Physics, University of Belgrade, Belgrade, Serbia

${ }^{b b b}$ Also at Università degli Studi di Siena, Siena, Italy

${ }^{c c c}$ Also at Trincomalee Campus, Eastern University, Sri Lanka

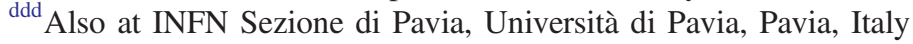

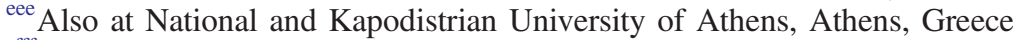

${ }^{\mathrm{fff}}$ Also at Universität Zürich, Zurich, Switzerland

${ }^{\text {ggg }}$ Also at Stefan Meyer Institute for Subatomic Physics, Vienna, Austria

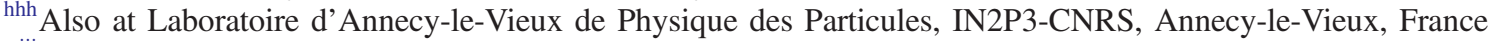

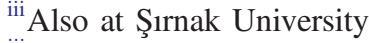

jij Also at Department of Physics, Tsinghua University, Beijing, China

${ }^{\mathrm{kkk}}$ Also at Near East University, Research Center of Experimental Health Science, Nicosia, Turkey

${ }^{111}$ Also at Beykent University, Istanbul, Turkey

${ }^{\mathrm{mmm}}$ Also at Istanbul Aydin University, Application and Research Center for Advanced Studies (App. \& Res. Cent. for Advanced Studies)

${ }^{\mathrm{nnn}}$ Also at Mersin University, Mersin, Turkey

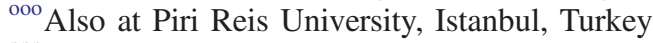

${ }^{\mathrm{ppp}}$ Also at Adiyaman University, Adiyaman, Turkey

${ }^{\mathrm{qqq}}$ Also at Ozyegin University, Istanbul, Turkey

${ }^{\text {rrr }}$ Also at Izmir Institute of Technology, Izmir, Turkey

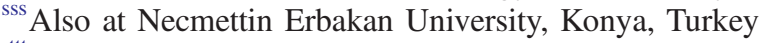

${ }^{\text {ttt }}$ Also at Bozok Universitetesi Rektörlügü

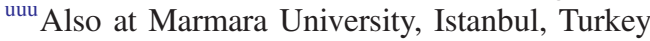

${ }^{\mathrm{vvv}}$ Also at Milli Savunma University

${ }^{w w w}$ Also at Kafkas University, Kars, Turkey

${ }^{\mathrm{xxx}}$ Also at Istanbul Bilgi University, Istanbul, Turkey

${ }^{\text {yyy }}$ Also at Hacettepe University, Ankara, Turkey

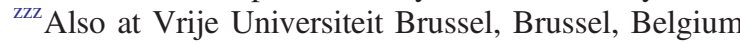

${ }^{\text {aaa }}$ Also at School of Physics and Astronomy, University of Southampton, Southampton, United Kingdom

${ }^{b b b b}$ Also at IPPP Durham University

${ }^{c c c c}$ Also at Monash University, Faculty of Science, Clayton, Australia

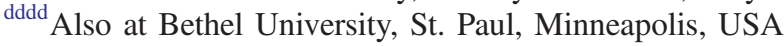

${ }^{\text {eee }}$ Also at Karamanoğlu Mehmetbey University, Karaman, Turkey

${ }^{\text {ffff }}$ Also at Bingol University, Bingol, Turkey

${ }^{g g g g}$ Also at Georgian Technical University, Tbilisi, Georgia

hhhh Also at Sinop University, Sinop, Turkey

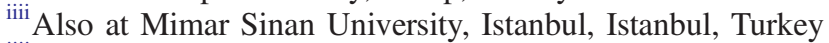

${ }^{j i j j}$ Also at Nanjing Normal University Department of Physics

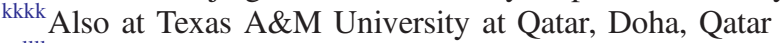

${ }^{1111}$ Also at Kyungpook National University, Daegu, Korea
} 